\title{
On broad Kaluza-Klein gluons
}

\author{
Rafel Escribano, ${ }^{a, b}$ Mikel Mendizabal, ${ }^{c}$ Mariano Quirós ${ }^{b}$ and Emilio Royo ${ }^{a, b}$ \\ ${ }^{a}$ Grup de Física Teòrica, Departament de Física, Universitat Autònoma de Barcelona, \\ 08193 Bellaterra (Barcelona), Spain \\ ${ }^{b}$ Institut de Física d'Altes Energies (IFAE), The Barcelona Institute of Science and Technology, \\ Campus UAB, 08193 Bellaterra (Barcelona), Spain \\ ${ }^{c}$ Deutsches Elektronen-Synchrotron, \\ 22607 Hamburg, Germany \\ E-mail: rescriba@ifae.es, mikel.mendizabal.morentin@desy.de, \\ quiros@ifae.es, eroyo@ifae.es
}

ABSTRACT: In theories with a warped extra dimension, composite fermions, as e.g. the right-handed top quark, can be very strongly coupled to Kaluza-Klein (KK) fields. In particular, the KK gluons in the presence of such composite fields become very broad resonances, thus remarkably modifying their experimental signatures. We have computed the pole mass and the pole width of the KK gluon, triggered by its interaction with quarks, as well as the prediction for proton-proton cross-sections using the full propagator and compared it with that obtained from the usual Breit-Wigner approximation. We compare both approaches, along with the existing experimental data from ATLAS and CMS, for the $t \bar{t}, t \bar{t} W, t \bar{t} Z, t \bar{t} H$, and $t t \overline{t t}$ channels. We have found differences between the two approaches of up to about $100 \%$, highlighting that the effect of broad resonances can be dramatic on present, and mainly future, experimental searches. The channel $t t \overline{t t}$ is particularly promising because the size of the cross-section signal is of the same order of magnitude as the Standard Model prediction, and future experimental analyses in this channel, especially for broad resonances, can shed light on the nature of possible physics beyond the Standard Model.

Keywords: Phenomenology of Field Theories in Higher Dimensions

ArXiv ePrint: 2102.11241 


\section{Contents}

1 Introduction 1

2 The model 3

3 Mass and width of the KK gluon $\quad 6$

3.1 Vacuum polarization and renormalization 6

3.2 Pole approach 8

3.3 On-shell approach $\quad 9$

3.4 Comparison between on-shell and pole approaches 10

4 Top-quark pair production at the LHC $\quad 11$

4.1 The partonic cross-section 11

$\begin{array}{lll}4.2 & \text { The proton cross-section } & 13\end{array}$

5 Top-quark pair plus $W, Z$ or $H$ production at the LHC 16

5.1 Top-quark pair plus $W$ production 16

$\begin{array}{lll}5.2 & \text { Top-quark pair plus } Z \text { production } & 18\end{array}$

$\begin{array}{lll}5.3 & \text { Top-quark pair plus Higgs production } & 19\end{array}$

6 Four top-quark production at the LHC 19

7 Conclusions $\quad 22$

\section{Introduction}

The solution to the problem of the Standard Model (SM) sensitivity to ultra-violet (UV) physics, also known as the hierarchy problem, generally implies new physics at the TeV scale. Two main solutions have been proposed thus far: supersymmetry [1], and a warped extra dimension [2]. ${ }^{1}$ The most strongly coupled and model independent fields in these theories are, respectively, the supersymmetric gluino $\tilde{g}$ (a spin 1/2 Majorana fermion) and the (first) Kaluza-Klein (KK) massive gluon $G$ (a spin 1 boson), with the same quantum numbers as the gluon, and mass around the TeV. Even though the spectra of particles in both theories are very rich, the production of these strongly coupled particles is considered as the smoking gun of the corresponding theories.

Elusiveness of experimental data at the LHC renders it relevant to reconsider, on more general grounds, the hierarchy problem. While gluinos in supersymmetric theories where $R$-parity is conserved appear in reactions as missing energy, e.g. $\tilde{g} \rightarrow q \tilde{q}$, where the gluino

\footnotetext{
${ }^{1}$ The latter is dual to a conformal field theory (CFT) or composite Higgs theory.
} 
mass cannot be reconstructed, present experimental searches on KK-gluon production focus on searching for (narrow) bumps on the invariant mass of its decay products, e.g. in $G \rightarrow q \bar{q}$.

As an attempt to explain the above lack of experimental evidence, theories where the KK gluons constitute a continuum above a mass gap, instead of isolated resonances, have recently been proposed [3-14], as well as unparticle models [15]. In this work, though, we want to explore a more conventional solution: the case of broad KK gluons, with mass $M$ and total width $\Gamma$. On one hand, as it is well known, given that KK gluons are generically strongly coupled to heavy SM fermions, the associated decay channels can naturally make them broad resonances. On the other hand, while production cross-sections decrease with increasing values of the mass $M$, for a fixed value of $M$ experimental bounds tend to deteriorate for larger values of the width $\Gamma$, or, in other words, for increasing values of the ratio

$$
r \equiv \Gamma / M,
$$

which leads to milder experimental bounds from direct searches.

In this work, we will concentrate in the production of the first KK mode $\left(G \equiv G^{(1)}\right)$ of gluons, as they are the most strongly coupled and model independent extra particles in the theory, and consider the effect of broad resonances by computing the pole masses and pole decay widths as the zeros of the inverse renormalized propagator; as it will be shown, this effect cannot be neglected for strongly coupled fields and broad resonances. We will also consider the minimal and most model independent case where only decay channels $G \rightarrow q \bar{q}$, where $q$ are the SM quarks, are considered, as the decay channel $G \rightarrow g g$ is forbidden.

By using MADGRAPH5_AMC [16], we will apply our results to present LHC bounds on $t \bar{t}$ as final state. Previous similar studies have concentrated on narrower $(r<0.2)$ resonances $[17,18]$, or tried to fit the large forward-backward (FB) asymmetry at the Tevatron using decay channels involving top-quark partners [19-21], or considered the case of KK resonances in the electroweak (model dependent) sector, which then allows lepton channels as possible final states $[22,23]$. We will also apply our theoretical results to the study of other final state channels, in particular to $t \bar{t} W, t \bar{t} Z, t \bar{t} H$, and $t t \bar{t} \bar{t}$, whose experimental detection is currently work in progress [24-29].

The present work is organized as follows. In section 2 we introduce the class of fivedimensional (5D) models, whose first KK gluon $G$ is considered in this work. In section 3 we compute the pole mass and pole width of the $G$ resonance by means of one-loop diagrams with propagating quarks, and compare the pole approach for the full propagator with the on-shell approach, which motivates the Breit-Wigner (BW) approximation. The topquark pair cross-section production $p p \rightarrow t \bar{t} X$ is studied in section 4 as a function of the renormalized mass for fixed values of the width, and as a function of the width for fixed values of the renormalized mass. In both cases we compare our predictions with the available experimental data from ATLAS. The results presented in this section attempt to motivate the use of the full propagator in the experimental analysis of broad resonances. In sections 5 and 6 , we compare our predictions for the channels $t \bar{t} W, t \bar{t} Z$ and $t \bar{t} H$, and $t t \overline{t t}$, as functions of the renormalized mass and width, with the existing experimental data. The present uncertainty of the experimental data does not allow to draw strong conclusions, albeit the $t t \overline{t t}$, where the SM prediction is of the same order of magnitude 
as the contribution from new physics, appears to be the most promising avenue for future experimental and theoretical endeavors. Finally, our conclusions are presented in section 7 .

\section{The model}

Our model is based on a 5D theory which contains a warped extra dimension $y$, with metric given as $d s^{2}=e^{-A(y)} \eta_{\mu \nu} d x^{\mu} d x^{\nu}-d y^{2}$ in proper coordinates, where $\eta_{\mu \nu}=$ $\operatorname{diag}(1,-1,-1,-1)$, and possessing ultra-violet (UV) and infra-red (IR) branes at $y=0$ and $y=y_{1}$, respectively. There is also a bulk propagating field $\phi$ which must stabilize the brane-to-brane distance and give a mass to the (otherwise massless) radion [30]. In fact, one can trade the fifth dimension $y$ by the value of the field $\phi^{2}$ by using the superpotential $W(\phi)[31]$, which is a function that characterizes the $5 \mathrm{D}$ gravitational metric as

$$
\frac{d A}{d \phi}=\frac{\kappa^{2}}{3}\left(\frac{\partial \log W}{\partial \phi}\right)^{-1}
$$

and gives rise to the bulk potential

$$
V(\phi)=\frac{1}{8}\left(\frac{\partial W}{\partial \phi}\right)^{2}-\frac{\kappa^{2}}{6} W^{2}(\phi)
$$

where $\kappa^{2}=1 /\left(2 M^{3}\right)$ and $M$ is the $5 \mathrm{D}$ Planck scale.

The metric originally proposed by Randall and Sundrum (RS) [2] was Anti-de Sitter in $5 \mathrm{D}\left(A d S_{5}\right)$ and, thus, characterized by a constant superpotential $W_{R S}=6 k / \kappa^{2}$, where $k$ is a constant of the order of the $4 \mathrm{D}$ Planck scale. This superpotential leads to the linear behavior $A_{R S}(y)=k y$ characteristic of an $A d S_{5}$ theory. It was soon realized that this theory was in conflict with electroweak precision observables, unless the mass of the first KK mode was $\gtrsim 10 \mathrm{TeV}$. This fact had an impact on the electroweak sector of the theory, which had to be modified to avoid the electroweak constraints. Two classes of solutions were advocated in the literature to prevent electroweak bounds.

One solution, proposed in ref. [32], was to enlarge the electroweak gauge sector in the bulk to $\mathrm{SU}(2)_{L} \otimes \mathrm{SU}(2)_{R} \otimes \mathrm{U}(1)_{B-L}$, which contains a custodial symmetry $\mathrm{SU}(2)_{V}$, and where $\mathrm{SU}(2)_{R} \otimes \mathrm{U}(1)_{B-L} \rightarrow \mathrm{U}(1)_{Y}$ is achieved on the UV brane by boundary conditions, while the whole group is unbroken on the IR brane, where custodial symmetry is intact. In this way, the SM gauge bosons $W_{L}^{a}, B$ have $(+,+)$ boundary condition on the (UV, IR) branes, and thus possess zero modes which correspond with the SM gauge bosons, while $W_{R}^{1,2}, Z_{R}$ have $(-,+)$ boundary conditions, and thus only with massive modes. ${ }^{3}$ In this case we can consider that the back-reaction of the field $\phi$ on the metric is negligible and then the superpotential $W(\phi) \simeq W_{R S}$. In this model, left-handed fermions are in $\mathrm{SU}(2)_{L}$ doublets and right-handed ones are arranged in $\mathrm{SU}(2)_{R}$ doublets while the Higgs is a fundamental bidoublet in the bulk. Furthermore, in this class of theories there are also (gauge-Higgs unification) models, with extended custodial gauge groups, for which the Higgs is the fifth

\footnotetext{
${ }^{2}$ Note that $\phi$ is fixed to values $\phi_{0}$ and $\phi_{1}$ by potentials localized in the UV and IR branes, respectively.

${ }^{3}$ These massive modes have been recently used to accommodate the $R_{D^{(*)}}$ anomalies [33].
} 
component $A_{5}$ of an odd gauge field $A_{\mu}$, which gets a mass from a radiative ColemanWeinberg mechanism. Now, the electroweak sector is a general gauge group $\mathcal{G}$ broken by boundary conditions to $\mathrm{SU}(2)_{L} \otimes \mathrm{U}(1)_{Y}$ at the UV brane and to the subgroup $\mathcal{H}$, which contains a custodial symmetry, on the IR brane. Here the Higgs lies along the fifth dimension of the coset group $\mathcal{G} / \mathcal{H}$, so that the choice of the groups $\mathcal{G}$ and $\mathcal{H}$ defines different models [3436]. In this general class of models, with an enlarged custodial symmetry in the electroweak sector, the color group $\mathrm{SU}(3)_{c}$ is unrelated to the electroweak symmetry breaking and thus the gluon sector is pretty much model independent, with KK gluons strongly coupled, as in the RS model, to composite fermions, localized toward the IR brane, as the top quark.

A different proposed solution is to use the stabilizing field $\phi$ in a regime where the back reaction on the gravitational metric is important near the IR brane and mild near the UV brane, thus, keeping the properties of RS theories to solve the hierarchy problem. In these theories, the back-reaction creates a naked singularity beyond the IR brane and they are dubbed soft-wall (SW) metric theories [6, 37-43]. Typically, theories with strong backreactions on the metric are characterized by exponential superpotentials, as e.g. $W_{S W}=$ $6 k / \kappa^{2}\left(1+e^{a \phi}\right)^{b}[44]$ with $a$ and $b$ real parameters, and a bulk gauge group $\mathrm{SU}(3)_{c} \otimes$ $\mathrm{SU}(2)_{L} \otimes \mathrm{U}(1)_{Y}$, which is just the SM gauge group. In this class of models, we can understand the improvement from the electroweak constraints by the fact that the physical Higgs boson profile, $\tilde{h}(y)=h(0) e^{\alpha k y-A(y)}$ with $\alpha>2$ to solve the hierarchy problem, unlike the case of the RS metric, is peaked away from the IR brane [40], while the KK modes are localized toward the IR brane, which makes their overlap small enough to cope with electroweak constraints for a choice of the model parameters. This analysis was performed in refs. [45, 46]. Again, in this class of theories as in custodial models, the color group is $\mathrm{SU}(3)_{c}$ and, thus, KK gluons are very much model independent.

In all previous models, the SM fermions are realized as chiral zero modes of $5 \mathrm{D}$ fermions, with localization along the fifth dimension determined by a 5D Dirac mass, conveniently chosen as $M_{f_{L, R}}=\mp c_{f_{L, R}} W(\phi)$, where the upper (lower) sign applies for a fermion with left-handed (right-handed) zero mode. With this convention, the fermion zero modes are localized near the UV (IR) brane for $c_{f_{L, R}}>1 / 2\left(c_{f_{L, R}}<1 / 2\right)$. Specifically, their wavefunctions in the fifth dimension are given by

$$
\Psi_{f_{L, R}}^{(0)}(x, y)=f_{L, R}(y) \psi_{L, R}(x), \quad f_{L, R}(y)=\frac{e^{\left(2-c_{L, R}\right) A(y)}}{\left[\int d y e^{\left(1-2 c_{L, R}\right) A}\right]^{1 / 2}}
$$

where $\psi_{L, R}(x)$ are the $4 \mathrm{D}$ wave functions. Moreover, after interaction with the Higgs field, the $4 \mathrm{D}$ mass of the fermion $f$ is determined by the constants $c_{f_{L}}$ and $c_{f_{R}}$, which in turn determine the overlapping integral with the Higgs wave function, along with the 5D Yukawa couplings. In this way, heavy (light) fermions are localized toward the IR (UV) brane and thus have constants $c_{f}<1 / 2\left(c_{f}>1 / 2\right)$. 


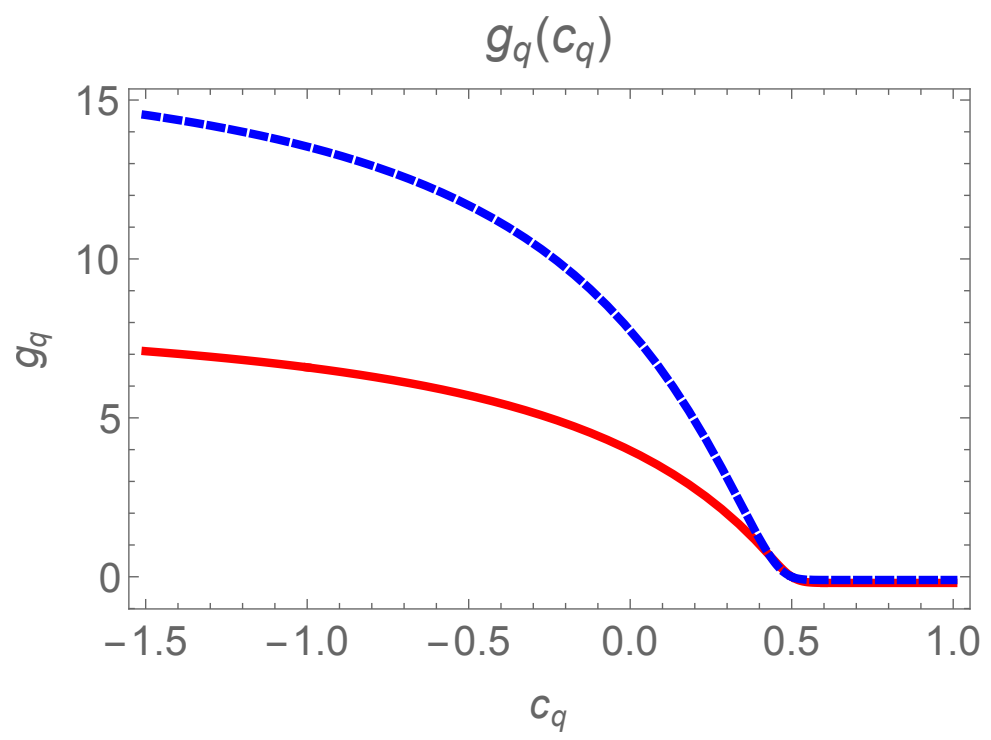

Figure 1. Coupling of the first gluon KK mode $G$ with the quark $q$ as a function of its localizing parameter $c_{q}$ for two models: the lower (solid) curve corresponds to the RS metric and the upper (dashed) curve to the SW metric for the choice of parameters $a=0.15$ and $b=2$.

Likewise, the coupling of the quark $q_{L, R}$ with the $\mathrm{KK}$ gluon $G^{(n)}$, with normalized profile $f_{G^{(n)}}$, is given by ${ }^{4}$

$$
g_{G^{(n)} q \bar{q}}=g_{s} g_{q}^{n}\left(c_{q}\right), \quad g_{q}^{n}\left(c_{q}\right) \equiv \int e^{-3 A} q^{2}(y) f_{G^{(n)}}(y) d y,
$$

where $g_{s}$ is the strong coupling constant of $\mathrm{SU}(3)_{c}$. As the KK modes $G^{(n)}$ are strongly localized toward the IR brane, it turns out that heavy quarks $\left(c_{q}<1 / 2\right)$ can be strongly coupled to $G^{(n)}$, while light quarks $\left(c_{q}>1 / 2\right)$ are weakly coupled.

In the present work, the focus revolves around the couplings of the first KK mode of the gluon (the lightest gluon KK mode) with quarks $q_{L, R}$. For illustrative purposes, the coupling $g_{q} \equiv g_{q}^{1}\left(c_{q}\right)$ is shown in figure 1 as a function of the localizing parameter $c_{q}$ for the two different class of models considered. The lower (solid) curve corresponds to the RS metric and its limiting value for $c_{q}<1 / 2,\left|c_{q}\right| \gg 1$, is $g_{q}=\sqrt{2 k y_{1}} \simeq 8.4$. In the other limit, when $c_{q}>1 / 2,\left|c_{q}\right| \gg 1$, the limiting behavior is $g_{q} \simeq-0.2$. The upper (dashed) curve corresponds to the considered class of SW models for particular values of the parameters $a=0.15, b=2$ for which the electroweak oblique observables $S$ and $T$ are minimized [45, 46]. In this case, one gets the behavior $g_{q} \simeq-0.2$ for UV localized quarks, while for quarks localized toward the IR we see that $g_{q} \simeq 15$. Needless to say, coupling values $\alpha_{q}=g_{q}^{2} / 4 \pi \gtrsim 4 \pi$ will give rise to non-perturbative behaviors and will make perturbative calculations absolutely untrustworthy. The plots in figure 1 illustrate two sort of extreme examples of 5D theories with a warped extra dimension. We expect that for arbitrary theories, the values of the coupling $g_{q}$ will fall in the region of values that appear in figure 1.

\footnotetext{
${ }^{4}$ Notice that, due to the different localization of quarks $q_{L}$ and $q_{R}$, their interaction with the KK modes $G^{(n)}$ is not vector-like, as it happens with its zero mode, the SM gluon $g \equiv G^{(0)}$.
} 
In this work, we consider the right-handed top $t_{R}$ as the most strongly coupled fermion so that $g_{t_{R}}$ is kept as a free parameter. On the other hand, in view of the strong constraints on the coupling of the left-handed bottom to the $Z$ boson, $\delta g_{Z b_{L} \bar{b}_{L}}=(0.33 \pm 0.17) \times$ $10^{-2}$ [47], and given that $g_{t_{L}}=g_{b_{L}}$ due to $\left(t_{L}, b_{L}\right)^{T}$ being part of an $\mathrm{SU}(2)_{L}$ doublet, we will fix $g_{t_{L}}=g_{b_{L}}=g_{b_{R}}=1$. In the absence of a UV theory for flavor, fixing the mass of the third generation quarks can be easily done with the help of 5D Yukawa couplings. Finally, we will localize the quarks $q_{L}$ and $q_{R}$ of the first and second generation $q=u, d, c, s$ near the UV brane, such that $g_{q_{L, R}} \simeq-0.2$.

In the next section, we will compute the total decay rate $\Gamma=\sum_{q} \Gamma(G \rightarrow q \bar{q})$ and we will see that it depends on the coupling $g_{t_{R}}$, so that we will trade the free parameter $g_{t_{R}}$ by the total width $\Gamma$. Moreover, as the coupling of $G$ with two gluons vanishes, by orthonormality of the wave functions, quarks are the only decay channels that we will consider in this work.

\section{Mass and width of the KK gluon}

In this section, we first calculate the heavy KK-gluon vacuum polarization (VP) at oneloop, associated to the effect of virtual quark-antiquark pairs of given chirality, and, next, resum its effect into the KK-gluon propagator. After that, we renormalize the propagator following two different approaches: the pole approach, where the renormalized VP function remains exact, and the on-shell approach, where this function is expanded around the KKgluon mass, and thus approximated by a Breit-Wigner function. Finally, we explain how to calculate the corresponding mass and width of the KK-gluon resonance in each approach and compare both sets of parameters.

\subsection{Vacuum polarization and renormalization}

The one-loop contribution from quarks $q=\left(\begin{array}{c}q_{L} \\ q_{R}\end{array}\right)$ to the KK-gluon VP, $\Pi_{\mu \nu}^{a b}\left(q^{2}\right)$, where $a, b=1, \ldots, 8$ are indices in the $\mathrm{SU}(3)$ Lie algebra, is written as

$$
i \Pi_{\mu \nu}^{a b}\left(q^{2}\right)=i \delta^{a b}\left[g_{\mu \nu} q^{2} \Pi\left(q^{2}\right)-q_{\mu} q_{\nu} \Delta\left(q^{2}\right)\right],
$$

with $\Pi\left(q^{2}\right)=\bar{\Pi}\left(q^{2}\right)+\delta M^{2} / q^{2}$ and

$$
\begin{aligned}
& \bar{\Pi}\left(q^{2}\right)=\Delta\left(q^{2}\right)+\frac{g_{s}^{2}}{4 \pi^{2}} \sum_{q} g_{q_{A}}^{2} \frac{m_{q}^{2}}{q^{2}} {\left[2+F\left(q^{2}\right)\right], } \\
& \Delta\left(q^{2}\right)=-\frac{g_{s}^{2}}{24 \pi^{2}} \sum_{q}\left(g_{q_{V}}^{2}+g_{q_{A}}^{2}\right) {\left[\frac{2}{\varepsilon}-\gamma_{E}+\ln 4 \pi+\ln \frac{\mu^{2}}{m_{q}^{2}}\right.} \\
&\left.+\frac{5}{3}\left(1+\frac{12 m_{q}^{2}}{5 q^{2}}\right)+\left(1+\frac{2 m_{q}^{2}}{q^{2}}\right) F\left(q^{2}\right)\right], \\
& \delta M^{2}=\frac{g_{s}^{2}}{4 \pi^{2}} \sum_{q} g_{q_{A}}^{2} m_{q}^{2}\left(\frac{2}{\varepsilon}-\gamma_{E}+\ln 4 \pi+\ln \frac{\mu^{2}}{m_{q}^{2}}\right),
\end{aligned}
$$


where $q^{2}$ is the squared KK-gluon momentum, $g_{s}$ is the QCD coupling constant with $\alpha_{s}=g_{s}^{2} / 4 \pi, g_{q_{V, A}} \equiv\left(g_{q_{L}} \pm g_{q_{R}}\right) / 2$ are the vector and axial KK-gluon-quark couplings, in units of $g_{s}$, defined in terms of the corresponding left- and right-handed ones, $\varepsilon$ the regulator of the ultraviolet divergence, $\gamma_{E}$ the Euler-Mascheroni constant, $\mu$ the arbitrary scale which appears in dimensional regularization, $m_{q}$ the quark mass, and $F\left(q^{2}\right)$ the loop associated function given by

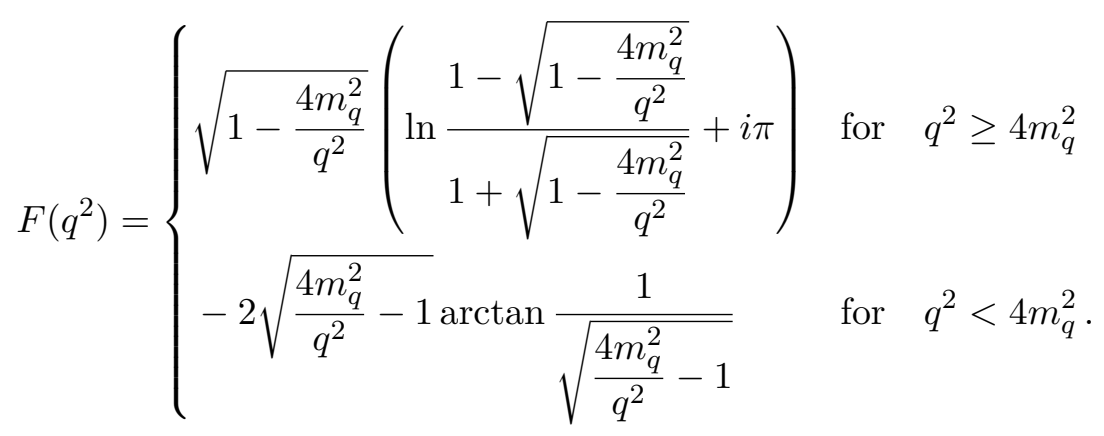

The correction of the former contribution to the KK-gluon propagator after resummation is given by

$$
\begin{aligned}
i G_{\mu \nu}^{a b} & =i G_{\mu \nu}^{(0) a b}+i G_{\mu \alpha}^{(0) a c} i \Pi_{c d}^{\alpha \beta}\left(q^{2}\right) i G_{\beta \nu}^{(0) d b}+\cdots \\
& =i \delta^{a b}\left[\frac{-g_{\mu \nu}+\frac{q_{\mu} q_{\nu}}{q^{2}}}{q^{2}-M_{0}^{2}}\left(1+\frac{q^{2} \Pi\left(q^{2}\right)}{q^{2}-M_{0}^{2}}\right)+\frac{q_{\mu} q_{\nu}}{q^{2}} \frac{1}{M_{0}^{2}} \text { terms }+\cdots\right] \\
& =i \delta^{a b}\left(\frac{-g_{\mu \nu}+\frac{q_{\mu} q_{\nu}}{q^{2}}}{q^{2}-M_{0}^{2}-q^{2} \Pi\left(q^{2}\right)}+\frac{q_{\mu} q_{\nu}}{q^{2}} \frac{1}{M_{0}^{2}} \text { terms }\right)
\end{aligned}
$$

where $i G_{\mu \nu}^{(0) a b}=i \delta^{a b} \frac{-g_{\mu \nu}+\frac{q_{\mu} q_{\nu}}{M_{0}^{2}}}{q^{2}-M_{0}^{2}}$ is the propagator at zeroth order in the unitary gauge and $M_{0}$ the KK-gluon bare mass. The $q_{\mu} q_{\nu}$ terms give rise to suppressed contributions in processes where the external particles have small masses compared to the KK-gluon mass, as is the case in the present work.

The correction of the KK-gluon propagator is divergent and must be renormalized. In order to regularize $\Pi\left(q^{2}\right)$ in eq. (3.2), one can define $\widehat{\Pi}\left(q^{2}\right) \equiv \bar{\Pi}\left(q^{2}\right)-\Re \bar{\Pi}\left(q_{0}^{2}\right)$ and thus

$$
\begin{aligned}
q^{2}-M_{0}^{2}-q^{2} \Pi\left(q^{2}\right) & =\left(q^{2}-M^{2}\right)\left[1-\Re \bar{\Pi}\left(q_{0}^{2}\right)\right]-q^{2} \widehat{\Pi}\left(q^{2}\right) \\
& =\left[1-\Re \bar{\Pi}\left(q_{0}^{2}\right)\right]\left[q^{2}-M^{2}-q^{2} \widehat{\Pi}\left(q^{2}\right)\right]+\mathcal{O}\left(g^{4}\right),
\end{aligned}
$$

where higher powers of the coupling constant are neglected. ${ }^{5}$ The parameter $M$ is an observable finite mass defined from $M_{0}^{2}+\delta M^{2} \equiv M^{2}\left[1-\Re \bar{\Pi}\left(q_{0}^{2}\right)\right]$, where $q_{0}$ is some chosen subtraction point used as the renormalization scale. ${ }^{6}$ The prefactor $1-\Re \bar{\Pi}\left(q_{0}^{2}\right)$ in front of the renormalized KK-gluon propagator is used for the renormalization of the coupling constant $g_{0}^{2} \equiv g^{2}\left[1-\Re \bar{\Pi}\left(q_{0}^{2}\right)\right]$.

\footnotetext{
${ }^{5}$ In the rest of this section, $g \equiv g_{s}$ is used for notational simplicity.

${ }^{6}$ The values of $M$ and $g$ depend on the chosen renormalization scale. For two different subtraction points $q_{0, i}$, with respective renormalized mass $M_{i}(i=1,2)$, we have $M_{2}^{2} / M_{1}^{2}=1+\Re \bar{\Pi}\left(q_{0,2}^{2}\right)-\Re \bar{\Pi}\left(q_{0,1}^{2}\right)+\mathcal{O}\left(g^{4}\right)$. The same goes for $g$.
} 
Bearing in mind that the KK-gluon propagator is attached to two vector minus axialvector currents weighted by the coupling constants, the net effect of renormalization for its transverse part is ${ }^{7}$

$$
\frac{g_{0}^{2}\left(-g_{\mu \nu}+\frac{q_{\mu} q_{\nu}}{q^{2}}\right)}{q^{2}-M_{0}^{2}-q^{2} \Pi\left(q^{2}\right)} \longrightarrow \frac{g^{2}\left(-g_{\mu \nu}+\frac{q_{\mu} q_{\nu}}{q^{2}}\right)}{q^{2}-M^{2}-q^{2} \widehat{\Pi}\left(q^{2}\right)}+\mathcal{O}\left(g^{6}\right),
$$

which from this point onwards is considered as the propagator to be used in our calculations.

\subsection{Pole approach}

Within the so-called pole approach framework [48-50], the renormalized propagator in eq. (3.6) does not have a pole at $q^{2}=M^{2}$. In the pole approach, the expression $D\left(q^{2}\right)=$ $q^{2}-M^{2}-q^{2} \widehat{\Pi}\left(q^{2}\right)$ is separated into its real and imaginary parts and is defined in the first Riemann sheet as $D_{\mathrm{I}}(s)=s-M^{2}-s\left[\Re \bar{\Pi}_{+}(s)-\Re \bar{\Pi}\left(q_{0}^{2}\right)\right]-i s \Im \bar{\Pi}_{+}(s)$, with $s=q^{2}$ and $\bar{\Pi}_{+}(s) \equiv \bar{\Pi}(s+i \epsilon)$ ( $\epsilon$ is an infinitesimal positive definite parameter). Due to the presence of the quark threshold, ${ }^{8}$ the complex function $\bar{\Pi}(s)$ is analytical everywhere in the complex plane with exception of a branch cut on the real $s$-axis starting at $4 m_{t}^{2}$ up to $+\infty$, the so-called unitary cut, which is associated to the bi-valued nature of the square root function. Accordingly, in addition to the physical sheet (the first Riemann sheet), there is an unphysical sheet (the second Riemann sheet) where the poles associated to resonances are found. The discontinuity across the cut is given by

$$
\begin{aligned}
\operatorname{Disc} \bar{\Pi}(s) & =\bar{\Pi}(s+i \epsilon)-\bar{\Pi}(s-i \epsilon) \\
& =\bar{\Pi}(s+i \epsilon)-\bar{\Pi}^{*}(s+i \epsilon)=2 i \Im \bar{\Pi}_{+}(s),
\end{aligned}
$$

where in the second equality the Schwarz reflection principle, $\bar{\Pi}^{*}(s+i \epsilon)=\bar{\Pi}(s-i \epsilon)$, has been used because the function is purely real below the threshold. The analytical continuation from the first to the second Riemann sheet is given by

$$
\begin{aligned}
\bar{\Pi}_{\mathrm{II}}(s+i \epsilon) & =\bar{\Pi}(s-i \epsilon)=\bar{\Pi}(s+i \epsilon)-\operatorname{Disc} \bar{\Pi}(s) \\
& =\Re \bar{\Pi}_{+}(s)-i \Im \bar{\Pi}_{+}(s) \equiv \bar{\Pi}_{-}(s) .
\end{aligned}
$$

In summary, the final form of the propagator's denominator $D(s)$ from which one ought to look for poles in the second Riemann sheet is

$$
D_{\mathrm{II}}(s)=s-M^{2}-s\left[\Re \bar{\Pi}_{+}(s)-\Re \bar{\Pi}\left(q_{0}^{2}\right)\right]+i s \Im \bar{\Pi}_{+}(s) .
$$

Since the function $\bar{\Pi}(s)$ also contains an implicit dependence on the modulus of the quark three-momentum, $p(s)=\sqrt{s-4 m^{2}} / 2$, the convention for defining the two different Riemann sheets is $(\mathrm{I}, \mathrm{II}) \equiv(+,-):(\Im p>0, \Im p<0)$ for the first and second sheets, respectively. In practice, one can move from one sheet to the other by replacing $p(s) \rightarrow-p(s)$

\footnotetext{
${ }^{7}$ The terms proportional to $q_{\mu} q_{\nu} / q^{2}$ in the longitudinal part of the propagator are also renormalized: $g_{0}^{2} / M_{0}^{2} \rightarrow g^{2} / M^{2}+\mathcal{O}\left(g^{4}\right)$.

${ }^{8}$ In the following, we consider the approximation that all quarks, excluding the top quark, are massless. This approximation is valid since $M \gg m_{q}$ for $q=u, d, s, c, b$. Due to this simplification, there is effectively only one quark threshold and it is located at $4 m_{t}^{2}$.
} 
in $D(s)$ and, therefore, $D_{\mathrm{I}}(s) \equiv D[s, p(s)]$ and $D_{\mathrm{II}}(s) \equiv D[s,-p(s)]$, as can be seen by inspecting eq. (3.2).

Once the parametric values of the KK-gluon mass $M$ and the KK-gluon-quark couplings (or $g_{t_{R}}$ in this simplified analysis ${ }^{9}$ ) are given, or in the future possibly extracted from LHC experimental data, the true resonance parameters, the so-called pole parameters, which are model- and process-independent, are obtained from the solution of the pole equation $D_{\mathrm{II}}\left(s_{\mathrm{p}}\right)=0$ with $s_{\mathrm{p}} \equiv M_{\mathrm{p}}^{2}-i M_{\mathrm{p}} \Gamma_{\mathrm{p}}$, where $M_{\mathrm{p}}$ and $\Gamma_{\mathrm{p}}$ are the pole mass and the pole width of the resonance. If for real $s, \bar{\Pi}_{+}(s)=\bar{\Re} \Pi_{+}(s)+i \Im \bar{\Pi}_{+}(s)$, then for arbitrary complex $s, \bar{\Pi}_{+}(s)=\Re \Re \bar{\Pi}_{+}(s)-\Im \Im \bar{\Pi}_{+}(s)+i\left[\Im \Re \bar{\Pi}_{+}(s)+\Re \Im \bar{\Pi}_{+}(s)\right]$, that is,

$$
\begin{aligned}
D_{\mathrm{II}}\left(s_{\mathrm{p}}\right)= & s_{\mathrm{p}}-M^{2}-s_{\mathrm{p}}\left[\Re \Re \bar{\Pi}_{+}\left(s_{\mathrm{p}}\right)-\Im \Im \bar{\Pi}_{+}\left(s_{\mathrm{p}}\right)-\Re \bar{\Pi}\left(q_{0}^{2}\right)\right] \\
& -i s_{\mathrm{p}}\left[\Im \Re \bar{\Pi}_{+}\left(s_{\mathrm{p}}\right)+\Re \Im \bar{\Pi}_{+}\left(s_{\mathrm{p}}\right)\right]=0 .
\end{aligned}
$$

\subsection{On-shell approach}

An alternative framework, the so-called on-shell approach [48-50], is based on an expansion of $\bar{\Pi}\left(q^{2}\right)$ around $q^{2}=M^{2}$ allowing the full propagator to have a pole at $q^{2}=M^{2}$ with some residue (field-strength renormalization) $\mathcal{Z}$. Taking only the real part of the propagator's denominator, ${ }^{10}$

$$
\begin{aligned}
q^{2}-M_{0}^{2}-q^{2} \Re \Pi\left(q^{2}\right) & =q^{2}-\left(M_{0}^{2}+\delta M^{2}\right)-q^{2} \Re \bar{\Pi}\left(q^{2}\right) \\
& =\left(q^{2}-M^{2}\right)\left[1-\Re \bar{\Pi}\left(M^{2}\right)-q^{2} \Re \bar{\Pi}^{\prime}\left(M^{2}\right)\right]+\mathcal{O}\left(q^{2}-M^{2}\right)^{2} \\
& \equiv\left(q^{2}-M^{2}\right) \mathcal{Z}^{-1}+\mathcal{O}\left(q^{2}-M^{2}\right)^{2},
\end{aligned}
$$

where $\left.\Re \bar{\Pi}^{\prime}\left(M^{2}\right) \equiv \frac{d \Re \bar{\Pi}\left(q^{2}\right)}{d q^{2}}\right|_{q^{2}=M^{2}}$ and $\mathcal{Z}^{-1}=1-\Re \bar{\Pi}\left(M^{2}\right)-M^{2} \Re \bar{\Pi}^{\prime}\left(M^{2}\right)$. When the imaginary part of $\Pi\left(q^{2}\right)$ is included, the final form of the relevant part of the propagator is written as

$$
\begin{aligned}
\frac{g_{0}^{2}\left(-g_{\mu \nu}+\frac{q_{\mu} q_{\nu}}{q^{2}}\right)}{q^{2}-M_{0}^{2}-q^{2} \Pi\left(q^{2}\right)} & \longrightarrow \frac{g^{2}\left[1-\Re \bar{\Pi}\left(M^{2}\right)\right] \mathcal{Z}}{q^{2}-M^{2}-i \mathcal{Z} q^{2} \Im \bar{\Pi}\left(q^{2}\right)}\left(-g_{\mu \nu}+\frac{q_{\mu} q_{\nu}}{q^{2}}\right) \\
& =\frac{g^{2}\left[1+M^{2} \Re \bar{\Pi}^{\prime}\left(M^{2}\right)\right]}{q^{2}-M^{2}-i q^{2} \Im \bar{\Pi}\left(q^{2}\right)}\left(-g_{\mu \nu}+\frac{q_{\mu} q_{\nu}}{q^{2}}\right)+\mathcal{O}\left(g^{6}\right) .
\end{aligned}
$$

One can easily check that the final expressions for the relevant part of the propagator appearing in eq. (3.6), with $q_{0}=M$, and eq. (3.12) are equivalent at $\mathcal{O}\left(q^{2}-M^{2}\right)^{2}$.

In the on-shell approach, the expression $q^{2}-M^{2}-i \mathcal{Z} q^{2} \Im \bar{\Pi}\left(q^{2}\right)$ closely resembles the relativistic Breit-Wigner (BW) formula $q^{2}-M^{2}+i M \Gamma$. If $q^{2} \Im \bar{\Pi}\left(q^{2}\right)$ is small, so that the resonance is narrow, one can approximate $q^{2} \Im \bar{\Pi}\left(q^{2}\right)$ as $M^{2} \Im \bar{\Pi}\left(M^{2}\right)$ over the width of the resonance. Then, one has precisely the BW form with the identification

$$
\Gamma=-\mathcal{Z} M \Im \bar{\Pi}\left(M^{2}\right)=-M \Im \bar{\Pi}\left(M^{2}\right)+\mathcal{O}\left(g^{4}\right) .
$$

For a broad resonance, as it may well be the case for the heavy KK gluon, the full energy dependence of $s \Im \bar{\Pi}(s)$ must be taken into account though.

\footnotetext{
${ }^{9}$ As stated before, all quark chiral couplings excluding the top right one are fixed to predetermined values. In this way, the analysis is performed just in terms of $M$ and $g_{t_{R}}$.

${ }^{10}$ In this approach, the subtraction point will be chosen to be $q_{0}=M$.
} 

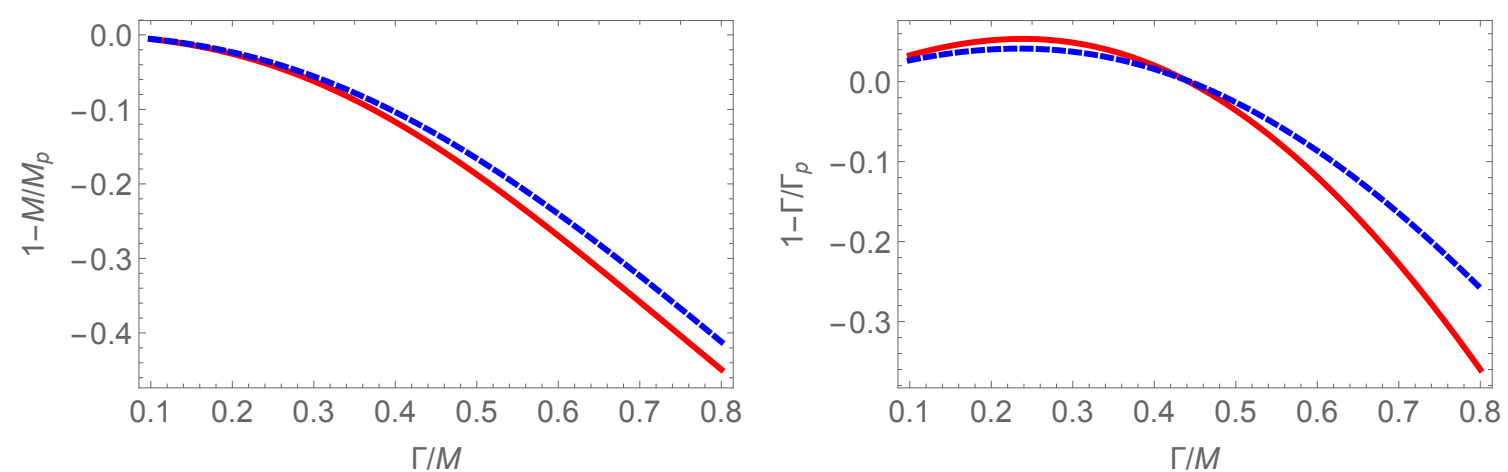

Figure 2. Plot of $1-M / M_{\mathrm{p}}$ (left panel) and $1-\Gamma / \Gamma_{\mathrm{p}}$ (right panel) as a function of the ratio $\Gamma / M$ for two values of the renormalized on-shell mass, $M=1 \mathrm{TeV}$ (solid red lines) and $5 \mathrm{TeV}$ (dashed blue lines).

\subsection{Comparison between on-shell and pole approaches}

In order to see the differences between the two approaches, let us numerically compare the values of the renormalized on-shell mass $M$ and width $\Gamma$ with those of the pole mass $M_{\mathrm{p}}$ and width $\Gamma_{\mathrm{p}}$ (at the subtraction point $q_{0}=M$ ) as a function of the right-handed top normalized coupling constant $g_{t_{R}}$ for different values of $M$. As an example, for the benchmark point $\left(M, g_{t_{R}}\right)=(2 \mathrm{TeV}, 5)$, in the on-shell approach one gets, based on eq. $(3.13),{ }^{11}$

$$
\begin{aligned}
\Gamma=\sum_{q} \Gamma(G \rightarrow q \bar{q})= & \frac{g_{s}^{2}}{24 \pi} M \sum_{q} \sqrt{1-\frac{4 m_{q}^{2}}{M^{2}}} \\
& \times\left[g_{q_{V}}^{2}\left(1+\frac{2 m_{q}^{2}}{M^{2}}\right)+g_{q_{A}}^{2}\left(1-\frac{4 m_{q}^{2}}{M^{2}}\right)\right]=0.371 \mathrm{TeV} .
\end{aligned}
$$

In the pole approach, using eq. (3.10) for the determination of the pole parameters in the second Riemann sheet of the complex plane, one gets $\left(M_{\mathrm{p}}, \Gamma_{\mathrm{p}}\right) \simeq(1.96,0.387) \mathrm{TeV}$ for the pole mass and width, respectively. If, instead of the relativistic definition $s_{\mathrm{p}} \equiv M_{\mathrm{p}}^{2}-i M_{\mathrm{p}} \Gamma_{\mathrm{p}}$, the non-relativistic inspired one $s_{\mathrm{p}}=\left(M_{\mathrm{p}}-i \Gamma_{\mathrm{p}} / 2\right)^{2}$ is used, the result is modified by less than five per mille. This modification is stable under changes in $M$ but grows as $g_{t_{R}}$ increases. For the highest values of the right-handed top coupling considered, $g_{t_{R}} \simeq 10$, the modification raises up to $6 \%$. In figure 2 , we plot the relative differences between the pole and renormalized on-shell masses (left panel), $1-M / M_{\mathrm{p}}$, and corresponding widths (right panel), $1-\Gamma / \Gamma_{\mathrm{p}}$, for the two values $M=1 \mathrm{TeV}$ and $5 \mathrm{TeV}$, as a function of the ratio $r \equiv$ $\Gamma / M$ in the range $r \in[0.1,0.8] .{ }^{12}$ As it can be seen, the renormalized on-shell mass is always bigger than the pole mass, $M>M_{\mathrm{p}}$, for any value of the ratio $r$, with the absolute maximum relative difference being around $45 \%(41 \%)$ at $r \simeq 0.8$ for $M=1(5) \mathrm{TeV}$, respectively.

\footnotetext{
${ }^{11}$ The value of the QCD coupling constant is taken to be $g_{s}\left(M_{Z}^{2}\right)=1.2172 \pm 0.0052$, or $\alpha_{s}\left(M_{Z}^{2}\right)=0.1179 \pm$ 0.0010, which leads after the renormalization group equation (RGE) running to $g_{s}\left(M^{2}\right)=1.027(0.985)$ for $M=2(5) \mathrm{TeV}$. Hereafter, for simplicity, we consider $g_{s}\left(M^{2}\right) \simeq 1$ in all numerical calculations.

${ }^{12}$ The corresponding right-handed top coupling $g_{t_{R}}$ can be obtained from eq. (3.14), once the renormalized on-shell mass $M$, the ratio $r$ and the rest of chirality quark couplings are fixed.
} 
Regarding the widths, the behavior depends upon the value of $r$ : for $r \lesssim 0.45$, the widths satisfy $\Gamma<\Gamma_{\mathrm{p}}$, reaching a difference of around 5\%(4\%) at $r \simeq 0.25$, while for $r \gtrsim 0.45$ one finds $\Gamma>\Gamma_{\mathrm{p}}$, reaching an absolute maximum difference of around $36 \%(26 \%)$ at $r \simeq 0.8$.

\section{Top-quark pair production at the LHC}

In the previous section, the on-shell and pole approaches have been compared in the construction of the heavy KK-gluon propagator. In this section, we consider the different effects of the two approaches in a measurable physical process, the top-quark pair production at the LHC, that is, the $p p \rightarrow t \bar{t} X$ reaction, mediated by the SM and the KK gluon $G$, where $X$ represents unobserved hadrons.

The hadronic cross-section can be written schematically as [51]

$$
\sigma(p p \rightarrow t \bar{t} X)=\int_{0}^{s} d \hat{s} \int_{\frac{1}{2} \log (\hat{s} / s)}^{\frac{1}{2} \log (s / \hat{s})} \frac{d y}{s} \sum_{a, b=q, \bar{q}, g} f_{a}^{(p)}\left(x_{1}\right) f_{b}^{(p)}\left(x_{2}\right) \hat{\sigma}(a b \rightarrow t \bar{t}),
$$

where $\hat{\sigma}(a b \rightarrow t \bar{t})$ is the partonic cross-section and $f_{a}^{(p)}\left(x_{1}\right) d x_{1}$ is the probability that a parton of type $a$ carries a fraction of the incident proton momentum that lies between $x_{1}$ and $x_{1}+d x_{1}$ (similarly for partons within the other incident proton). Moreover, $\hat{s}$ is the squared invariant mass of the $t \bar{t}$ system, $\hat{s}=x_{1} x_{2} s$, where $s=(13 \mathrm{TeV})^{2}$ is the squared center-of-mass energy of the proton-proton system, $x_{1,2}=\sqrt{\hat{s} / s} e^{ \pm y}$, and $y$ is the rapidity of the top-quark pair.

To illustrate the effects described in the previous section, we first discuss the partonic cross-section $q \bar{q} \rightarrow G^{*} \rightarrow t \bar{t}$ for $q \neq t .{ }^{13}$

\subsection{The partonic cross-section}

The cross-section of the Drell-Yan process at the partonic level $q \bar{q} \rightarrow G^{*} \rightarrow t \bar{t}$ is given by

$$
\begin{aligned}
\hat{\sigma}\left(q \bar{q} \rightarrow G^{*} \rightarrow t \bar{t}\right)= & \frac{g_{s}^{4}(M)}{54 \pi} \frac{\hat{s}}{|D(\hat{s})|^{2}} \sqrt{1-\frac{4 m_{t}^{2}}{\hat{s}}} \\
& \times\left(g_{q_{V}}^{2}+g_{q_{A}}^{2}\right)\left[g_{t_{V}}^{2}\left(1+\frac{2 m_{t}^{2}}{\hat{s}}\right)+g_{t_{A}}^{2}\left(1-\frac{4 m_{t}^{2}}{\hat{s}}\right)\right],
\end{aligned}
$$

where two different versions of the KK-gluon propagator denominator, $D(\hat{s})$, are to be assessed

$$
\begin{cases}\text { Full propagator: } & D_{\text {full }}(\hat{s})=\hat{s}-M^{2}-\hat{s} \widehat{\Pi}(\hat{s}), \\ \text { BW propagator: } & D_{\text {os }}(\hat{s})=\hat{s}-M^{2}+i M \Gamma .\end{cases}
$$

In figure 3, we present, as an example, the partonic cross-sections for the case of a KK gluon with a renormalized on-shell mass of $M=2 \mathrm{TeV}$ and two different widths $\Gamma$. Specifically, on the left panel, a relatively narrow heavy gluon with $\Gamma=200 \mathrm{GeV}$, i.e. $r=0.1$, is shown and, on the right panel, a broad one with $\Gamma=1.6 \mathrm{TeV}$, i.e. $r=0.8$, is displayed. In

\footnotetext{
${ }^{13}$ The case $a, b=g$ does not contribute to the KK-gluon cross-section since the vertex $g g G$ vanishes.
} 

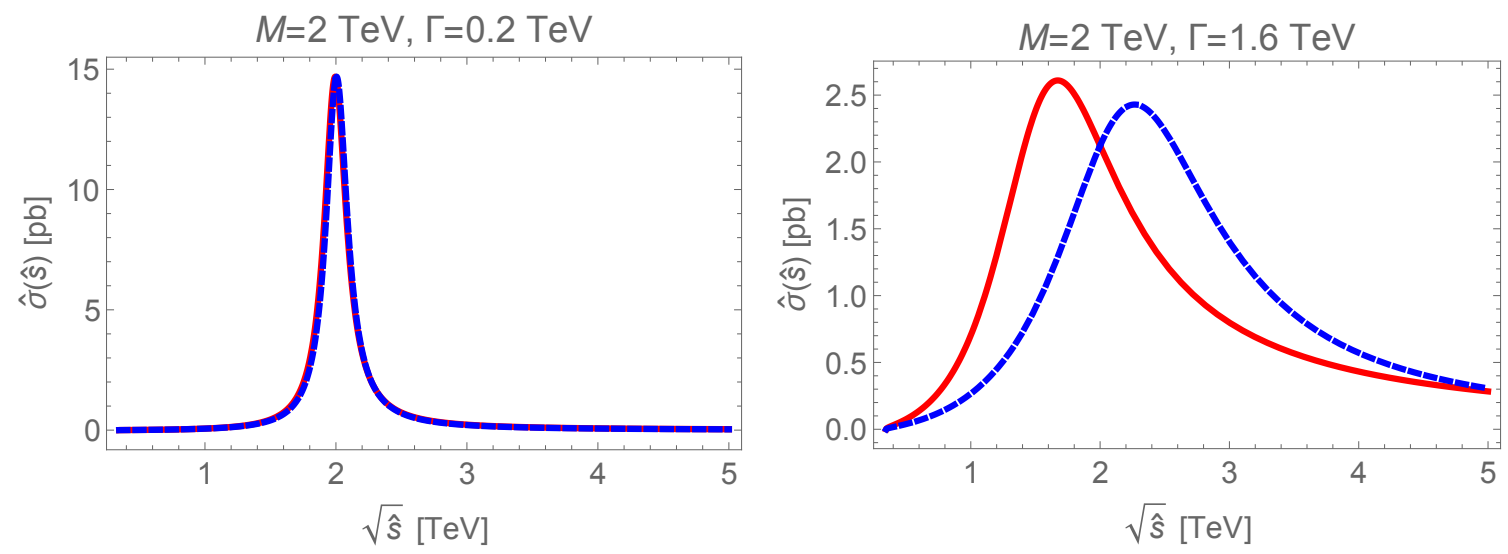

Figure 3. $q \bar{q} \rightarrow G^{*} \rightarrow t \bar{t}$ partonic cross-section as a function of the invariant mass of the topquark pair for a $\mathrm{KK}$ gluon of $M=2 \mathrm{TeV}$ with a "narrow" width of $\Gamma=200 \mathrm{GeV}$ (left panel) and a "broad" width of $\Gamma=1.6 \mathrm{TeV}$ (right panel), respectively. The red solid lines correspond to the full propagator and the blue dashed lines to the BW propagator with on-shell parameters.

both instances, we employ the two versions of the propagator (cf. eq. (4.3)), i.e. the full propagator $D_{\text {full }}$ (red solid lines) and the BW propagator with on-shell parameters $D_{\text {os }}$ (blue dashed lines). As one can clearly see, the two cross-sections are indistinguishable for narrow resonances, while, for broad ones, the location and height of the peaks are different, as well as their effective width. This is explained as follows. In the narrow case, the pole parameters associated to $(M, \Gamma)=(2,0.2) \mathrm{TeV}$ are $\left(M_{\mathrm{p}}, \Gamma_{\mathrm{p}}\right)=(1.989,0.206) \mathrm{TeV}$, thus making no substantial difference. In the broad case, however, $(M, \Gamma)=(2,1.6) \mathrm{TeV}$ and the corresponding pole parameters are found to be $\left(M_{\mathrm{p}}, \Gamma_{\mathrm{p}}\right)=(1.405,1.256) \mathrm{TeV}$; thus, $\left|D_{\text {full }}(\hat{s})\right|^{-1}$ peaks roughly at the pole mass, with a larger height and narrower shape than $\left|D_{\mathrm{os}}(\hat{s})\right|^{-1}$ (which, in turn, is located at the on-shell mass), as a result of the pole width being smaller compared to its on-shell counterpart. In the limit $m_{t}^{2} / \hat{s} \rightarrow 0$, which is a very good approximation, the partonic cross-section is proportional to $\hat{s} /|D(\hat{s})|^{2}$; as a consequence, the two peaks are displaced to higher values of the top-antitop invariant mass, keeping their relative distance approximately constant, and their corresponding heights are made more alike.

As explained in section 3.2, the resonance pole position for a specific parametrization of the propagator is obtained from $D_{\mathrm{II}}\left(s_{\mathrm{p}}\right)=0$, with $s_{\mathrm{p}}=M_{\mathrm{p}}^{2}-i M_{\mathrm{p}} \Gamma_{\mathrm{p}}$. For the on-shell approach, the pole parameters are trivially identified as $\left.\left(M_{\mathrm{p}}, \Gamma_{\mathrm{p}}\right)\right|_{\text {os }}=(M, \Gamma)$, while for the pole approach they are extracted from eq. (3.10). Using, again, the examples of narrow and broad resonances from above, the pole positions in each approach are, respectively, $\left.\left(M_{\mathrm{p}}, \Gamma_{\mathrm{p}}\right)\right|_{\mathrm{os}}=(2,0.2) \mathrm{TeV}$ and $\left.\left(M_{\mathrm{p}}, \Gamma_{\mathrm{p}}\right)\right|_{\text {pole }}=(1.989,0.206) \mathrm{TeV}$ for $(M, \Gamma)=(2,0.2) \mathrm{TeV}$, and $\left.\left(M_{\mathrm{p}}, \Gamma_{\mathrm{p}}\right)\right|_{\mathrm{os}}=(2,1.6) \mathrm{TeV}$ and $\left.\left(M_{\mathrm{p}}, \Gamma_{\mathrm{p}}\right)\right|_{\mathrm{pole}}=(1.405,1.256) \mathrm{TeV}$ for $(M, \Gamma)=(2,1.6) \mathrm{TeV}$. Since the pole position in the complex plane identifies a resonance with its mass and width, we see that, for the same renormalized $(M, \Gamma)$ values, one can obtain two different sets of pole parameters, and, therefore, allegedly two different KK gluons depending on the propagator parametrization employed. This, clearly, is nonsense: the pole position of the KK gluon is unique and, thus, independent of the parametriza- 

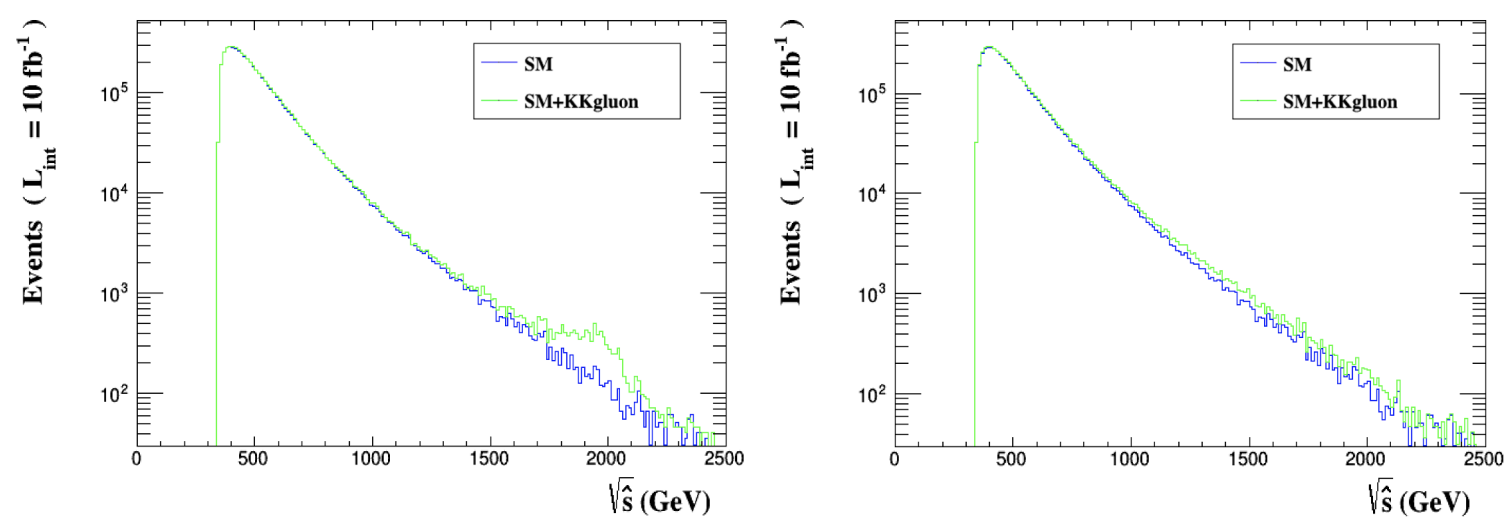

Figure 4. $p p \rightarrow t \bar{t}$ integrated cross-section as a function of the top-antitop invariant mass $\sqrt{\hat{s}}$ for $r=0.1$ (left panel) and $r=0.8$ (right panel). In both cases, the renormalized on-shell mass is set to $M=2 \mathrm{TeV}$. The number of distributed events is $10^{6}$ and the integrated luminosity is assumed to be $10 \mathrm{fb}^{-1}$. The blue lines correspond to the SM contribution alone and the green lines to the SM and KK-gluon contributions.

tion used. Similarly, if one instead fixes $\left(M_{\mathrm{p}}, \Gamma_{\mathrm{p}}\right)$, then two different sets of KK-gluon cross-section results can be obtained depending on the chosen propagator parametrization that would then need to be confronted with the available experimental data. Given this situation, we strongly recommend the use of the pole approach, that is, the full propagator, as it is the most complete and appropriate parametrization of the resonance. The on-shell approach, based on a BW description of the resonance, which is considered the simplest and, therefore, easiest to implement, is only a valid approximation for narrow resonances which are far from other resonances and thresholds; accordingly, we strongly discourage using it, specially in the context of the present work.

\subsection{The proton cross-section}

In this subsection, the cross-section for top-quark pair production in proton-proton collisions via an off-shell KK gluon produced in the $s$-channel, $p p \rightarrow G^{*} \rightarrow t \bar{t}$, is computed for several values of the renormalized mass $M$ and the ratio $r$, eq. (1.1), using MADGRAPH5_AMC [16]. We provide predictions for the two different propagator parametrizations discussed above, that is, the BW propagator with on-shell parameters and the full propagator, and compare our results with experimental data when available.

First of all, and in order to illustrate the effect of broad resonances on experimental detection, we display in figure 4 the proton-proton integrated cross-section as a function of the top-antitop invariant mass $\sqrt{\hat{s}}$ for $r=0.1$ (left panel) and $r=0.8$ (right panel). In both cases, the renormalized on-shell mass is set to $M=2 \mathrm{TeV}$. The number of distributed events is $10^{6}$ and the integrated luminosity is assumed to be $10 \mathrm{fb}^{-1}$. The calculation performed by MADGRAPH5_AMC [16] of both the SM background and the KK-gluon signal is at leading order. The blue lines correspond to the SM contribution alone and the green lines to the SM and KK-gluon contributions, where the KK-gluon contributions are calculated using the full propagator. As shown, for $r=0.1$, or equivalently $\Gamma=200 \mathrm{GeV}$, 

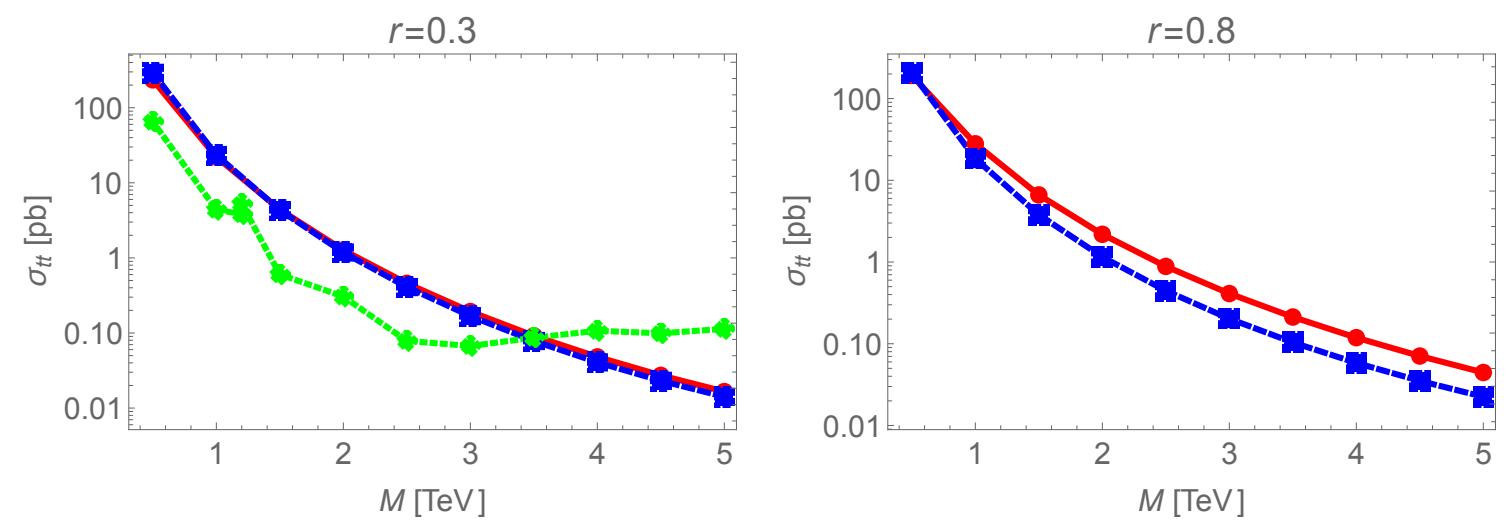

Figure 5. $p p \rightarrow G^{*} \rightarrow t \bar{t}$ integrated cross-section as a function of the renormalized on-shell mass $M$ for the ratio $r=0.3$ (left panel) and $r=0.8$ (right panel). The red solid lines correspond to the full propagator, the blue dashed lines to the BW propagator with on-shell parameters, and the green dotted line to the experimental 95\% CL upper limit observed by the ATLAS collaboration [52].

the effect of the narrow KK gluon is clearly seen as a bump at $\sqrt{\hat{s}} \simeq 2 \mathrm{TeV}$ standing up over the SM background. This behavior makes it easier to isolate the signal and allows for the discovery of such a narrow resonance. On the contrary, for $r=0.8$, corresponding to a broad $\mathrm{KK}$ gluon with $\Gamma=1.6 \mathrm{TeV}$, the bump disappears and its effect is spread in a much wider region of $\sqrt{\hat{s}}$, making it more difficult to extract a possible signal from the background. Once again, we want to stress that figure 4 is for illustrative purpose only.

In figure 5, we display the proton-proton integrated cross-section as a function of $M$ for $r=0.3$ (left panel) and $r=0.8$ (right panel). The red solid lines correspond to the predictions based on the full propagator, while the blue dashed ones are based on the BW propagator with on-shell parameters. The cross-sections are calculated for the same values as the ATLAS collaboration in ref. [52], namely, from $M=0.5 \mathrm{TeV}$ to $5 \mathrm{TeV}$ in steps of $0.5 \mathrm{TeV}$. All the values shown are computed from simulations with $10^{5}$ events. As expected, the predicted values decrease when the KK-gluon on-shell mass increases.

For the $r=0.3$ case, the predictions for the two types of propagators are almost indistinguishable, the reason being the low value of the KK-gluon on-shell width $\Gamma$ with respect to its mass $M$, a behaviour already anticipated at the partonic level. These two sets of predictions are compared with the experimental 95\% CL upper limits observed by the ATLAS collaboration in ref. [52] (green dotted line). Note that the two experimental values for the cross-section at $M=1.2 \mathrm{TeV}$ correspond to the resolved and boosted analysis methods taken by ATLAS. Our values using the BW propagator are very similar to the theoretical prediction displayed in figure 12 of ref. [52] by the red line. As it can be seen in figure 5 , values of the KK-gluon on-shell mass $\lesssim 3.5 \mathrm{TeV}$ for a resonance with $r=0.3$ are excluded at the $95 \%$ CL for both propagator parametrizations.

For the $r=0.8$ case, the two sets of predictions are clearly distinguishable, with the cross-section values from the full propagator being always bigger than those of the BW propagator for all values of $M$. There is a simple explanation for this behavior: as we have already discussed before, the pole mass is smaller than the renormalized one, 

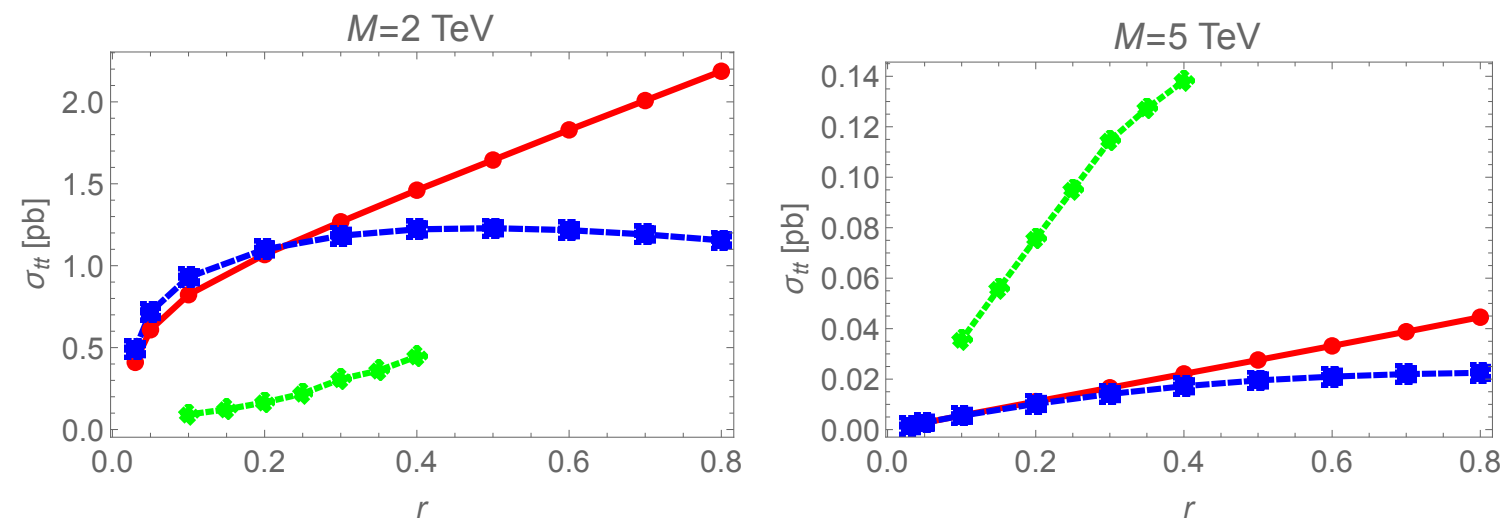

Figure 6. $p p \rightarrow G^{*} \rightarrow t \bar{t}$ integrated cross-section as a function of the ratio $r=\Gamma / M$ for the renormalized on-shell mass $M=2 \mathrm{TeV}$ (left panel) and $5 \mathrm{TeV}$ (right panel). The red solid lines correspond to the full propagator, the blue dashed lines to the BW propagator with on-shell parameters, and the green dotted line to the experimental 95\% CL upper limit observed by the ATLAS collaboration [52].

$M_{\mathrm{p}}<M$; consequently, the maximum of the partonic cross-section computed using the full propagator takes place at a $\sqrt{\hat{s}}$ value that is smaller than the corresponding one for the BW propagator (cf. figure 3). At the proton level, a resonance with mass $M_{\mathrm{p}}$ is thus more copiously produced than a resonance with a larger mass $M$, and, consequently, the cross-section computed with the full propagator is larger than the cross-section obtained with the BW propagator, in agreement with both panels in figure 5. In addition, and as we saw in figure 2, the larger the value of $r$, the smaller the $M_{\mathrm{p}} / M$ ratio, which in turn leads to a larger ratio of cross-sections, $\sigma_{t \bar{t}}^{\text {full }} / \sigma_{t \bar{t}}^{\mathrm{BW}}$. This explains the difference between both panels in figure 5. Unfortunately, there currently is no experimental data for $r=0.8$ to compare with our results. Notwithstanding this, one can see that an experimental exclusion line would exclude smaller values of $M$ in the case of the BW propagator as compared to the full propagator. In fact, if one assumes an approximately flat crossing, then the difference would be of order $0.5 \mathrm{TeV}$ for $M \geq 1.5 \mathrm{TeV}$.

Similarly, in figure 6 we display the proton-proton integrated cross-section as a function of $r$ for $M=2 \mathrm{TeV}$ (left panel) and $5 \mathrm{TeV}$ (right panel). The cross-sections are calculated for values of $r$ ranging from $r=0.1$ to $r=0.8$, while the experimental ATLAS values [52] range from $r=0.1$ to $r=0.4$. We also include predicted values for $r=0.03$ and $r=0.05$ to test the convergence of both approaches for very narrow resonances. Using the full propagator, the predicted cross-sections increase as $r$, or equivalently $\Gamma$, increases; this is the case for the two values of $M$ analyzed here. Contrary to this, when the BW propagator is used, the predicted cross-sections appear to approach a constant value for $r \gtrsim 0.4$. Qualitatively, the results from figure 6 can be explained by the fact that the pole mass is always smaller than the renormalized one, $M_{\mathrm{p}}<M$, and, as already discussed above, the larger $r$ is, the smaller the ratio $M_{\mathrm{p}} / M$ becomes (cf. figure 2). This means that the ratio of cross-sections computed with full and BW propagators, $\sigma_{t \bar{t}}^{\text {full }} / \sigma_{t \bar{t}}^{\mathrm{BW}}$, increases for larger values of $r$, in agreement with both panels in figure 6 . Furthermore, the left panel in figure 2 also shows that the ratio $M_{\mathrm{p}} / M$ decreases for increasing values of $M$, which helps explain 
the difference between the panels in figure 6 . In view of the experimental exclusion lines shown in figure 6 , a KK gluon with an on-shell mass of $M=2 \mathrm{TeV}$ is excluded, at least for any $r \leq 0.4$. Conversely, a KK gluon with an on-shell mass of $M=5 \mathrm{TeV}$ is allowed for any width. This is in line with the conclusions obtained from the left panel in figure 5 whereby a possible exclusion starts at $M \simeq 3.5 \mathrm{TeV}$ for relatively narrow $(r=0.3) \mathrm{KK}$ gluons.

\section{Top-quark pair plus $W, Z$ or $H$ production at the LHC}

In the following subsections, we present our predictions for the total proton-proton crosssections into a top-antitop quark pair plus $W, Z$ or $H$ production at the LHC for different values of the KK-gluon on-shell mass $M$ and the ratio $r$. All the predicted values are computed with simulations using $10^{5}$ events and presented using $\mu_{\text {th }} \equiv \sigma_{\text {total }} / \sigma_{\text {SM }}$ defined as the ratio of the total cross-section with respect to the SM value. These results are compared, process by process, with available experimental data from the ATLAS or CMS collaborations in the form of $\mu_{\mathrm{obs}} \equiv \sigma_{\mathrm{obs}} / \sigma_{\mathrm{SM}}$, where $\sigma_{\text {obs }}$ is the observed cross-section. The calculated total cross-sections include the contributions of the SM, KK gluon and their interference.

For the SM contribution, we assume the same value the experimental collaboration with which we compare uses, while for the KK-gluon and the interference ${ }^{14}$ contributions we use the values given by MADGRAPH5_AMC [16]. Given that for each process we take the value of the SM cross-section provided by the experimental collaborations, the most conservative criterion followed to choose the data to compare with is the one offering a value of $\mu_{\mathrm{obs}}$ closer to unity, that is, more compatible with the SM. For the sake of simplicity, we only compare with the experimental data the predictions that we compute using the full propagator (pole approach), which is the most correct way to proceed. For the three processes that we analyze, we include plots of the ratio $\mu_{\mathrm{th}}$ as a function of $M$ for fixed values of $r=0.3$ and 0.8 , and $\mu_{\text {th }}$ as a function of $r$ for fixed $M=2 \mathrm{TeV}$ and $5 \mathrm{TeV}$. Our predictions are shown in solid (red) and blue (dashed) lines while the experimental data are displayed using bands corresponding to $1 \sigma$ (light green) and $2 \sigma$ (light yellow) deviations from the $\mu_{\text {obs }}$ (black dotted line) central values. A subset of representative Feynman diagrams contributing to each process is also shown for completeness.

\subsection{Top-quark pair plus $W$ production}

The $p p \rightarrow t \bar{t} W$ total cross-section involves a total of 76 Feynman diagrams, 8 of which involve a KK gluon. An example of such diagrams is shown in figure 7. The experimental observed ratios are $\mu_{t \bar{t} W}^{\mathrm{obs}}=1.39_{-0.16}^{+0.17}$ (for a reference $\mathrm{SM}$ value of $\sigma_{t \bar{t} W}^{\mathrm{SM}}=727 \mathrm{fb}$ ) measured by the ATLAS collaboration [27] and $\mu_{t \bar{t} W}^{\mathrm{obs}}=1.43 \pm 0.21$ (with $\sigma_{t \bar{t} W}^{\mathrm{SM}}=650 \mathrm{fb}$ ) by the CMS collaboration [26]. Both measurements exhibit a mild $\sim 2 \sigma$ departure from the SM prediction. This represents a small anomaly with respect to the SM that, if confirmed by future, more precise, data, should point toward the presence of new physics. Unexpectedly, the measurements of the observable $\mu_{t t W}^{\mathrm{obs}}$ from both experimental collaborations, ATLAS

\footnotetext{
${ }^{14}$ The interference is calculated by subtracting from the total cross-section the sum of the SM and KKgluon cross-sections.
} 


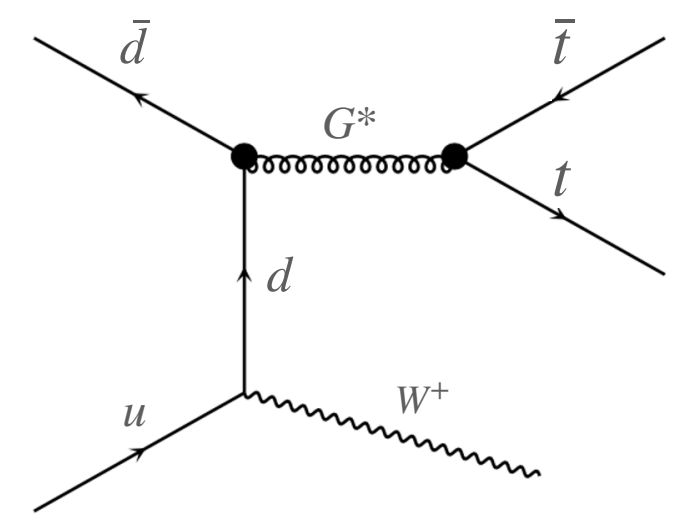

Figure 7. A representative Feynman diagram of the process $p p \rightarrow t \bar{t} W$ mediated by a KK gluon. This is only one of the 8 diagrams containing a KK gluon out of a total of 76 involved in the process.
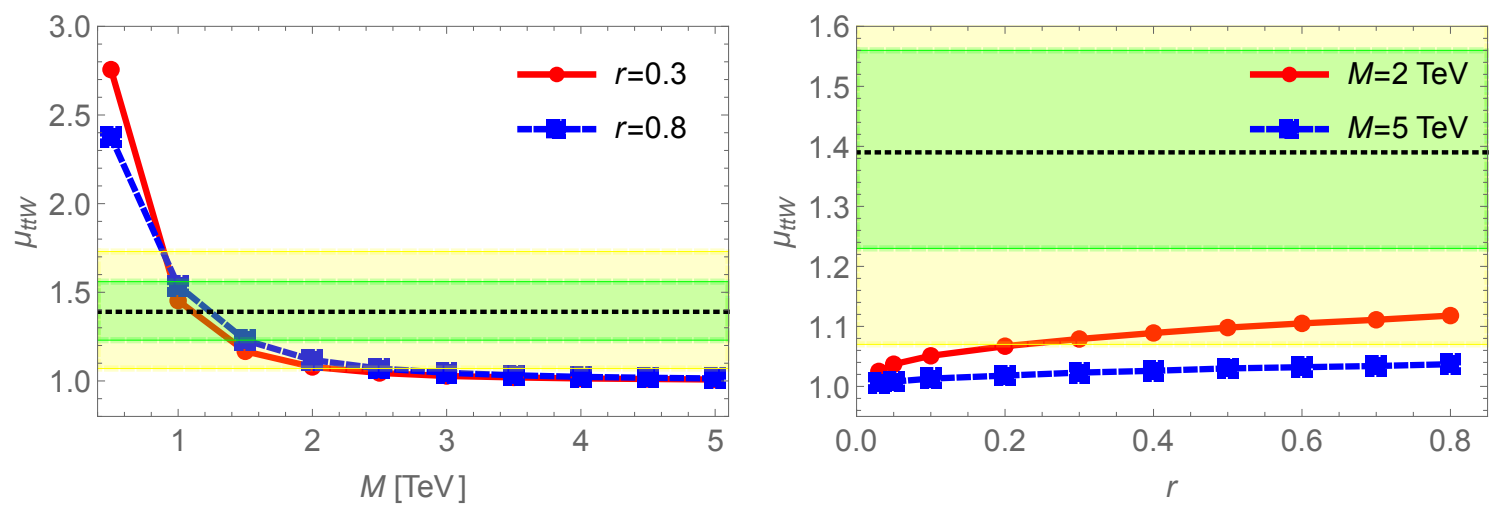

Figure 8. Ratio of the total cross-section $p p \rightarrow t \bar{t} W$ with respect to the SM value, $\mu_{t \bar{t} W}^{\mathrm{th}}$, as a function of the renormalized on-shell mass $M$ (left panel) for the fixed ratios $r=0.3$ (red solid line) and $r=0.8$ (blue dashed line), and as a function of the ratio $r$ (right panel) for the fixed masses $M=2 \mathrm{TeV}$ (red solid line) and $M=5 \mathrm{TeV}$ (blue dashed line). The different lines correspond to the predictions based on the full propagator (pole approach), while the bands represent $1 \sigma$ (light green) and $2 \sigma$ (light yellow) deviations from the $\mu_{t \bar{t} W}^{\mathrm{obs}}$ central value (black dotted line) observed by the ATLAS collaboration [27].

and CMS, are consistent with each other within $1 \sigma$. However, we prefer to conservatively wait for future confirmation or otherwise.

In figure 8, our predictions for $\mu_{t t W}^{\mathrm{th}}$ as a function of $M$ (left panel) and of the ratio $r$ (right panel) are compared with the experimental value observed by ATLAS. Should this experimental value be confirmed, then a KK gluon of mass roughly between 1 and $1.5 \mathrm{TeV}$ may be allowed at the $1 \sigma$ level, regardless of its width. However, if the bands are relaxed to include $2 \sigma$ deviations, the allowed range encompasses from about 0.8 to $2.5 \mathrm{TeV}$. As well as this, KK-gluon masses of 2 and $5 \mathrm{TeV}$ would be excluded at $1 \sigma$ irrespective of their widths, while at $2 \sigma$ only a $\mathrm{KK}$ gluon of $M=2 \mathrm{TeV}$ and $\Gamma \gtrsim 400 \mathrm{GeV}$ would be allowed. These results ought to be understood in conjunction and compared with the existing data on top-pair production, which yield, as we have seen in the previous section, the bound $M \gtrsim 3.5 \mathrm{TeV}$ for $r \leq 0.4$ at $95 \%$ CL. Specifically, a KK gluon with a mass $M=5 \mathrm{TeV}$, irrespective of $r$, should be excluded from the results presented in figure 8 . 

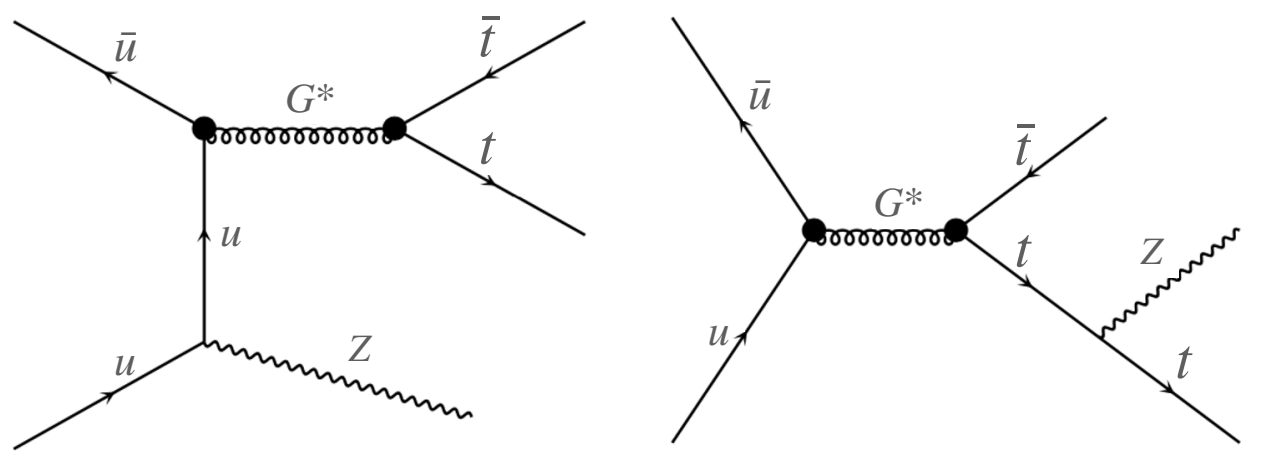

Figure 9. A representative subset of Feynman diagrams of the process $p p \rightarrow t \bar{t} Z$ mediated by a KK gluon. These are only two of the 16 diagrams containing a KK gluon out of a total of 116 involved in the process.
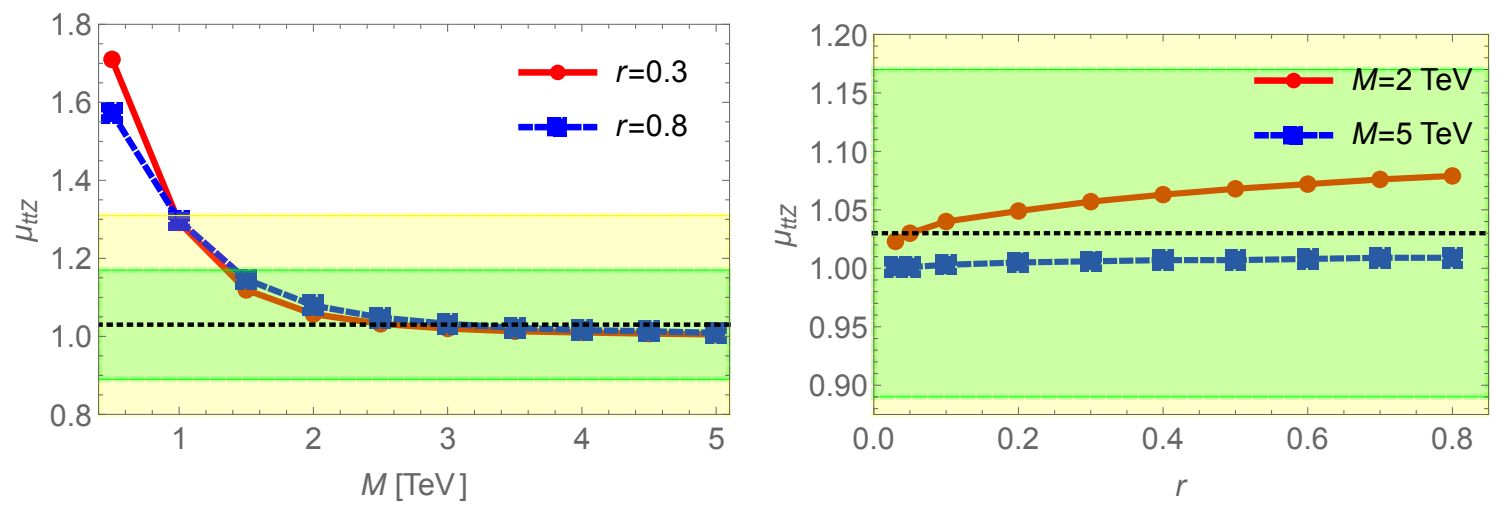

Figure 10. Ratio of the total cross-section $p p \rightarrow t \bar{t} Z$ with respect to the SM value, $\mu_{t \bar{t} Z}^{\text {th }}$, as a function of the renormalized on-shell mass $M$ (left panel) for the fixed ratios $r=0.3$ (red solid line) and $r=0.8$ (blue dashed line), and as a function of the ratio $r$ (right panel) for the fixed masses $M=2 \mathrm{TeV}$ (red solid line) and $M=5 \mathrm{TeV}$ (blue dashed line). The different lines correspond to the predictions based on the full propagator (pole approach), while the bands represent $1 \sigma$ (light green) and $2 \sigma$ (light yellow) deviations from the $\mu_{t \bar{t} Z}^{\text {obs }}$ central value (black dotted line) observed by the CMS collaboration [26].

\subsection{Top-quark pair plus $Z$ production}

The $p p \rightarrow t \bar{t} Z$ total cross-section involves a total of 116 Feynman diagrams, 16 of which involve a KK gluon. Examples of such diagrams are shown in figure 9. The KK-gluon mass can be reconstructed either from the $t \bar{t}$ invariant mass (left) or the $t \bar{t} Z$ one (right). The experimental observed ratios are $\mu_{t \bar{t} Z}^{\mathrm{obs}}=1.19 \pm 0.12$ (for a reference SM value of $\sigma_{t \bar{t} Z}^{\mathrm{SM}}=880 \mathrm{fb}$ ) measured by the ATLAS collaboration [29] and $\mu_{t \bar{t} Z}^{\mathrm{obs}}=1.03 \pm 0.14$ (with $\sigma_{t \bar{t} Z}^{\mathrm{SM}}=839 \mathrm{fb}$ ) by the CMS collaboration [26].

As it can be seen in figure 10 (left panel), and subject to experimental confirmation, a KK gluon of mass roughly below $1.4 \mathrm{TeV}$ is excluded at $1 \sigma$ for $r=0.3$, while for $r=0.8$ the exclusion rules out $M \lesssim 1.5 \mathrm{TeV}$. If one allows for $2 \sigma$ deviations, then only KKgluon masses below $1 \mathrm{TeV}$ are excluded. In addition, $\mathrm{KK}$ gluons of masses 2 and $5 \mathrm{TeV}$ are allowed at present (right panel) regardless of its widths. The experimental results from the 

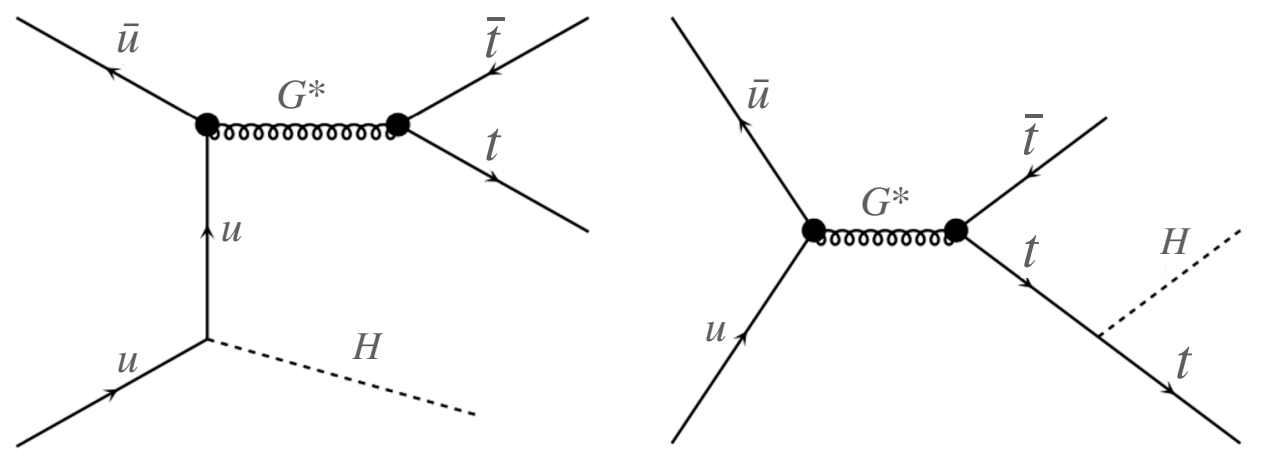

Figure 11. A representative subset of Feynman diagrams of the process $p p \rightarrow t \bar{t} H$ mediated by a KK gluon. These are only two of the 16 diagrams containing a KK gluon out of a total of 116 involved in the process.

$p p \rightarrow t \bar{t} Z$ channel are in very good agreement with the SM predictions and the excluded region of light KK-gluon masses was already excluded from the more robust results on topquark pair production in section 4 . It is worth highlighting that the $p p \rightarrow t \bar{t} Z$ results are consistent with a heavy mass KK gluon, in contradiction with the analysis of the $p p \rightarrow t \bar{t} W$ cross-section values in section 5.2.

\subsection{Top-quark pair plus Higgs production}

The $p p \rightarrow t \bar{t} H$ total cross-section involves the same 116 Feynman diagrams as the $t \bar{t} Z$ channel, of course, replacing the $Z$ by the $H$ (see figure 11). Similarly, the KK-gluon mass can be reconstructed either from the $t \bar{t}$ or the $t \bar{t} H$ invariant masses. The experimental observed ratios are $\mu_{t \bar{t} H}^{\mathrm{obs}}=0.70_{-0.33}^{+0.36}$ measured by the ATLAS collaboration [27] and $\mu_{t \bar{t} H}^{\mathrm{obs}}=$ $0.92_{-0.23}^{+0.25}$ by the CMS collaboration [26] (both with a reference SM value of $\sigma_{t \bar{t} H}^{\mathrm{SM}}=507 \mathrm{fb}$ ).

As is shown in figure 12 (left panel), and again subject to experimental verification, a KK gluon of mass roughly below $1.6 \mathrm{TeV}$ is excluded at $1 \sigma$ for $r=0.3$, while for $r=0.8$ any $M \lesssim 1.8 \mathrm{TeV}$ is ruled out. At $2 \sigma$, all mass values are allowed with exception of $M \simeq 1 \mathrm{TeV}$. The results from this subsection are consistent with the ones from the analysis of $t \bar{t} Z$ production but in tension with the outcome from $t \bar{t} W$ production. In summary, the production of $p p \rightarrow t \bar{t} H$ is in perfect agreement with the SM predictions, still with large uncertainties, and therefore it does not add any relevant information to the possible existence and properties of the heavy KK gluon.

\section{Four top-quark production at the LHC}

The four top-quark production at the LHC is, as of now, just a recently explored area by experimental collaborations $[24,25]$ due to its technical difficulties. This channel offers, in general, a new window for new physics searches and, in particular, allows for the hunting of a possible existing KK gluon. In this section, we compute the total cross-section $\sigma_{t t \overline{t t}}=\sigma(p p \rightarrow t t \overline{t \bar{t}})$, including the SM and KK-gluon contributions, for different values of the KK-gluon on-shell mass $M$ and the ratio $r$. In order to perform an accurate comparison with experimental data, we use for the SM cross-section the value given in ref. [53], that is 

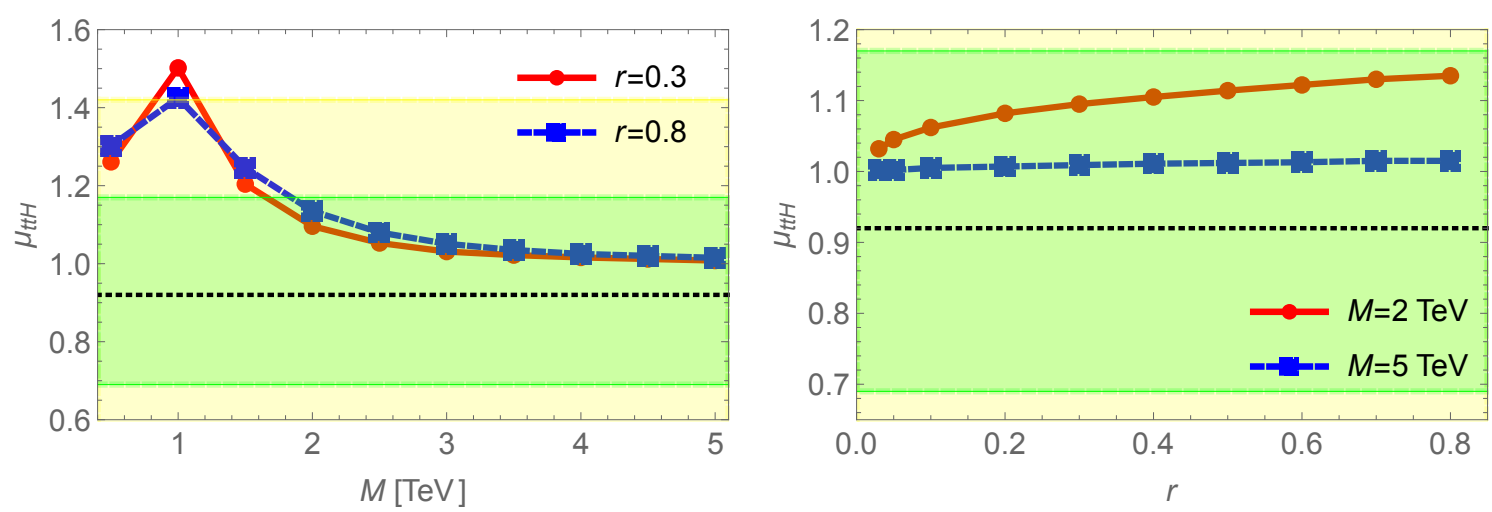

Figure 12. Ratio of the total cross-section $p p \rightarrow t \bar{t} H$ with respect to the SM value, $\mu_{t \bar{t} H}^{\mathrm{th}}$, as a function of the renormalized on-shell mass $M$ (left panel) for the fixed ratios $r=0.3$ (red solid line) and $r=0.8$ (blue dashed line), and as a function of the ratio $r$ (right panel) for the fixed masses $M=2 \mathrm{TeV}$ (red solid line) and $M=5 \mathrm{TeV}$ (blue dashed line). The different lines correspond to the predictions based on the full propagator (pole approach), while the bands represent $1 \sigma$ (light green) and $2 \sigma$ (light yellow) deviations from the $\mu_{t t \bar{t}}^{\mathrm{obs}}$ central value (black dotted line) observed by the CMS collaboration [26].

$\sigma_{t t t t}^{\mathrm{SM}}=12 \mathrm{fb}$, which is calculated at complete next-to-leading order, and consider it as our reference value. The contributions of the KK gluon and the interference of the KK gluon with the SM, both computed at leading order, are obtained using MADGRAPH5_AMC [16] by subtracting the SM cross-section from the total cross-section. Finally, our final predicted values for the total cross-section are the sum of the reference SM cross-section from experiment plus the KK-gluon and interference cross-sections computed in MADGRAPH5_AMC with simulations containing $10^{5}$ events.

The $p p \rightarrow t t \overline{t \bar{t}}$ total cross-section at leading order involves a total of 1920 Feynman diagrams, 544 of which involve a KK gluon. A subset of representative diagrams entering this process is shown in figure 13. As was done in the previous section, we only compare with the experimental data our predictions based on the use of the full propagator (pole approach). For this process, we include plots of $\sigma_{t t \overline{t t}}$ as a function of $M$ for fixed $r=0.3$ and 0.8 , and as a function of $r$ for fixed $M=2 \mathrm{TeV}$ and $5 \mathrm{TeV}$. Our predictions are shown in red (solid) and blue (dashed) lines, while the observed cross-section is displayed using bands corresponding to $1 \sigma$ (light green) and $2 \sigma$ (light yellow) deviations from the $\sigma_{t t t t t}^{\text {obs }}$ (black dotted line) central value. From the two experimental cross-sections, $\sigma_{t t \overline{t t}}^{\text {obs }}=24_{-6}^{+7} \mathrm{fb}$ measured by the ATLAS collaboration [25] and $\sigma_{t t t t t}^{\text {obs }}=9.4_{-5.6}^{+6.2} \mathrm{fb}$ by the CMS collaboration [24], we choose for comparison the latter as it is closer to the reference SM cross-section.

In figure 14 (left panel), the total $p p \rightarrow t t \overline{t t}$ cross-section is shown as a function of $M$ for $r=0.3$ (red solid line) and $r=0.8$ (blue dashed line). As it can be seen, a KK gluon of $M \lesssim 4 \mathrm{TeV}$ is excluded for $r=0.3$ at the $1 \sigma \mathrm{CL}$, whereas for $r=0.8$ all the values of $M$ are excluded. At $2 \sigma$, the exclusion limits are relaxed to $M \lesssim 2.8 \mathrm{TeV}$ for $r=0.3$ and $M \lesssim 4.6 \mathrm{TeV}$ for $r=0.8$. In the first case, the exclusion limit seems to be in reasonable agreement with the one obtained from the top-quark pair cross-section, $M \lesssim 3.5 \mathrm{TeV}$ for $r=0.3$. The right panel of figure 14 displays the total $p p \rightarrow t t \overline{t \bar{t}}$ cross-section as a function 

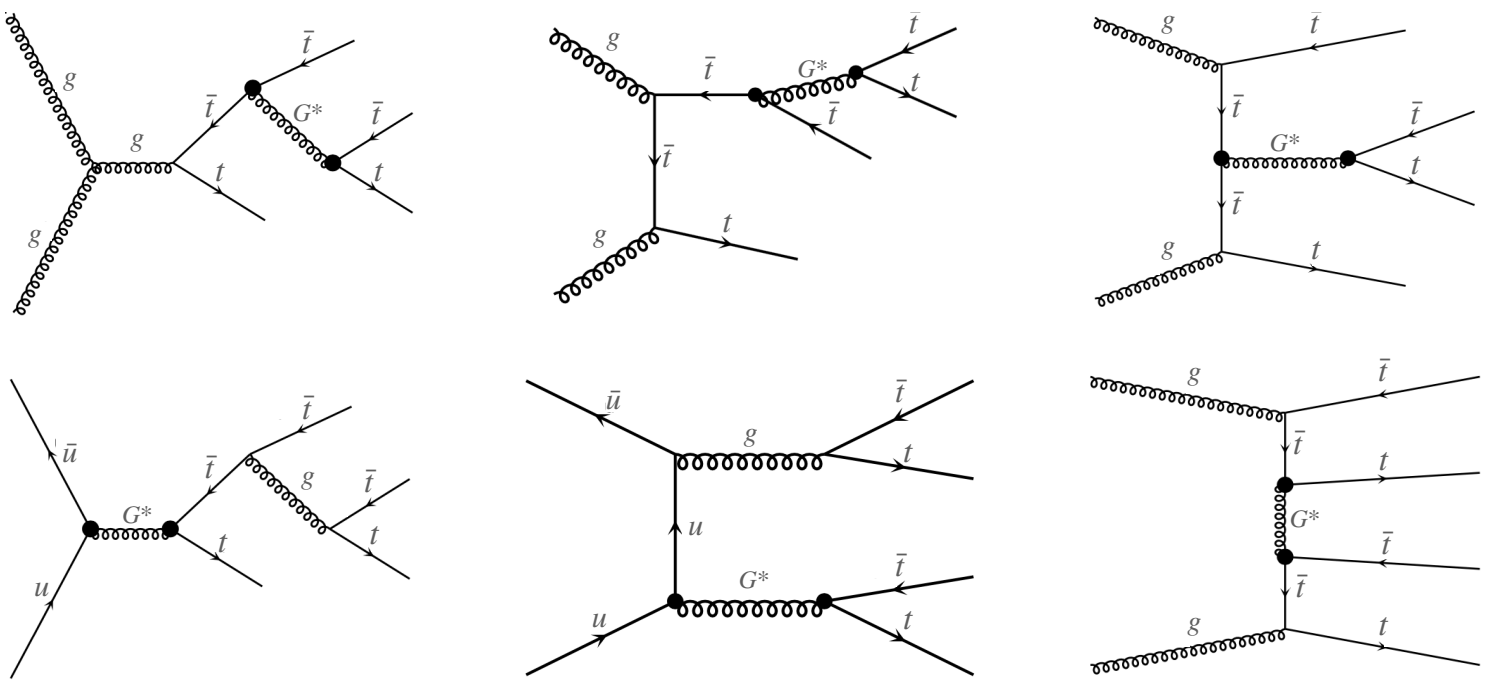

Figure 13. A representative subset of Feynman diagrams of the process $p p \rightarrow t t \overline{t t}$ mediated by a KK gluon. These are only 6 of the 544 diagrams containing a single KK gluon out of a total of 1920 involved in the process.
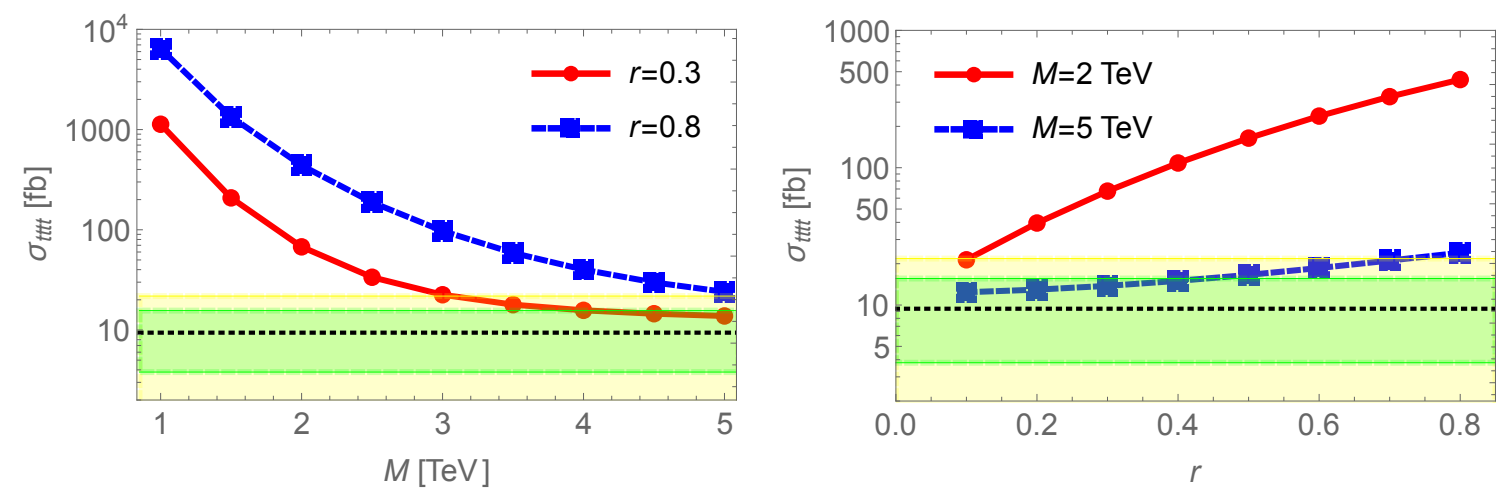

Figure 14. The total cross-section $p p \rightarrow t t \overline{t t}$ as a function of the renormalized on-shell mass $M$ (left panel) for the fixed ratios $r=0.3$ (red solid line) and $r=0.8$ (blue dashed line), and as a function of the ratio $r$ (right panel) for the fixed masses $M=2 \mathrm{TeV}$ (red solid line) and $M=5 \mathrm{TeV}$ (blue dashed line). The different lines correspond to the predictions based on the full propagator (pole approach), while the bands represent $1 \sigma$ (light green) and $2 \sigma$ (light yellow) deviations from the $\sigma_{t t \bar{t} t}^{\text {obs }}$ central value (black dotted line) observed by the CMS collaboration [24].

of $r$ for $M=2 \mathrm{TeV}$ (red solid line) and $M=5 \mathrm{TeV}$ (blue dashed line). Clearly, a KK gluon of $M=2 \mathrm{TeV}$ is excluded regardless of its width at the $1 \sigma \mathrm{CL}$, while for $M=5 \mathrm{TeV}$ the exclusion limit rules out any $r \gtrsim 0.4$ or $\Gamma \gtrsim 2 \mathrm{TeV}$. At the $2 \sigma$ level, only a very narrow KK gluon with $r \simeq 0.1$ or $\Gamma \simeq 200 \mathrm{GeV}$ is allowed for the $M=2 \mathrm{TeV}$ case, while for the $M=5 \mathrm{TeV}$ case the exclusion limits discard any $r \gtrsim 0.7$ or $\Gamma \gtrsim 3.5 \mathrm{TeV}$.

In summary, the $p p \rightarrow t t \overline{t t}$ channel seems the most promising one, as the SM prediction and signal are of similar size. For the moment, though, our simplified analysis appears to exclude, at the $2 \sigma$ level, resonances with $M \lesssim 4.6 \mathrm{TeV}$ for $r=0.8$, and with $r \gtrsim 0.7$ for $M=5 \mathrm{TeV}$, both regions being allowed by the present top-pair production 
experimental data (cf. section 4). It appears from the present analysis that future data on the $p p \rightarrow t t \overline{t t}$ channel shall be most relevant for uncovering new physics and, in particular, for detecting the presence of heavy KK gluons, a remnant of warped theories aiming to solve the hierarchy problem.

\section{Conclusions}

In theories with a warped extra dimension and two branes, i.e. the UV and the IR, the Higgs field must be localized toward the IR brane in order to solve the hierarchy problem. In these theories, where all Standard Model fields are propagating in the bulk of the extra dimension, Kaluza-Klein excitations are also localized toward the IR brane and interact strongly with all IR localized fields, or composite fields in the language of the dual 4D holographic theory. Moreover, the flavor problem in the quark sector can be solved if heavy quarks are very composite, and light quarks elementary. In this way, the most composite fermion ought to be the right-handed top quark $t_{R}$, which then can interact very strongly with the Kaluza-Klein states. Out of these Kaluza-Klein states, the gluons are the most model independent ones, as they do not interfere with the electroweak symmetry breaking phenomenon, and thus its phenomenology is, to a very large extent, model independent. Accordingly, the Kaluza-Klein gluon can decay mainly into the top-quark pair and get a broad width, which can have consequences at the phenomenological and experimental level.

In this work, we have studied the case of the first Kaluza-Klein gluon resonance with a width that mainly depends on the coupling to the quark $t_{R}$, which, in turn, depends on its particular localization in the extra dimension. We have found that the strong coupling with the quark sector has two effects: on one hand, as we have described, it can produce a broad resonance which can easily jeopardize experimental capabilities of detection; on the other hand, it can have a strong renormalization effect on its mass, which can also strongly change its production rate at LHC. Both effects are taken into account by computing the pole mass $M_{\mathrm{p}}$ and pole width $\Gamma_{\mathrm{p}}$ as the full propagator pole in the second Riemann sheet of the complex s-plane, defined as $s_{\mathrm{p}}=M_{\mathrm{p}}^{2}-i M_{\mathrm{p}} \Gamma_{\mathrm{p}}$. We have then compared, for the $t \bar{t}$ channel, the predictions of the full propagator with that of the commonly used Breit-Wigner approximation, using renormalized values for the mass $M$ and width $\Gamma$. Admittedly, the latter is relatively accurate only for the case of narrow resonances.

The comparison of pole and renormalized quantities yield meaningful differences, depending, of course, on the strength of the coupling of the Kaluza-Klein gluon to the quark $t_{R}$. The main qualitative results are:

- The pole mass $M_{\mathrm{p}}$ is smaller than the renormalized mass $M$ by as much as $40 \%$. This has a great impact in the production cross-sections for the different processes we have analyzed.

- For small couplings, the pole width $\Gamma_{\mathrm{p}}$ is of the order of the renormalized one $\Gamma$; however, for large couplings, $\Gamma_{p}$ is smaller than $\Gamma$ by as much as $30 \%$.

We have computed with MADGRAPH5_AMC the cross-section of the $p p \rightarrow t \bar{t}$ process using the full propagator and the Breit-Wigner approximation, and compared the results. 
This has been done for different values of the renormalized mass $1 \mathrm{TeV}<M<5 \mathrm{TeV}$ and width $\Gamma / M<0.8$. As well as this, a comparison between our theoretical results for this channel with the available experimental data from the ATLAS collaboration has been performed. In addition, we have calculated cross-sections for the processes $t \bar{t} W, t \bar{t} Z, t \bar{t} H$, and $t t \overline{t t}$, and, again, compared with available empirical data from the ATLAS and CMS collaborations. In particular, we have found differences of order $100 \%$ for the case of broad resonances in the $t \bar{t}$ channel cross-sections, depending on whether the full or the BreitWigner propagator was employed. As for the $t \bar{t} W, t \bar{t} Z, t \bar{t} H$, and $t t \bar{t} \bar{t}$ channels, given that the experimental data are not accurate enough at present, we have just presented results using the full propagator for completeness. Once again, the theoretical predictions depend dramatically on the width of the resonance. We have found particularly interesting the $t t \overline{t t}$ channel for which the Standard Model prediction and the corrections from KaluzaKlein gluons are essentially of the same order of magnitude. Once again, the theoretical predictions in this channel can vary by more than one order of magnitude between narrow and large resonances.

Broader resonances are more strongly coupled (in this model, the strength of the coupling is related to the width of the resonance) and pole masses associated to these resonances turn out to be significantly smaller than the corresponding renormalized ones, which, in turn, implies that they are more copiously produced. This has a remarkable impact on the experimental signature. Unfortunately, at present, experimental searches do not cover the case of very broad resonances. We hope, however, that future experimental endeavors will consider this scenario so that strong experimental bounds on resonance masses and widths can be extracted, and robust comparison with the presently analyzed theoretical model's predictions can be performed. This will decisively point out the capabilities and limitations of the theory.

\section{Acknowledgments}

We would like to thank A. Juste and M. Szewc for having participated in the early stages of this work, and E. Megias for discussions. This work is supported by the Secretaria d'Universitats i Recerca del Departament d'Empresa i Coneixement de la Generalitat de Catalunya under the grant 2017SGR1069, by the Ministerio de Economía, Industria y Competitividad under grants FPA2017-88915-P and FPA2017-86989-P, from the Centro de Excelencia Severo Ochoa under the grant SEV-2016-0588 and from the EU STRONG2020 project under the program H2020-INFRAIA-2018-1, grant agreement No. 824093. IFAE is partially funded by the CERCA program of the Generalitat de Catalunya.

Open Access. This article is distributed under the terms of the Creative Commons Attribution License (CC-BY 4.0), which permits any use, distribution and reproduction in any medium, provided the original author(s) and source are credited. 


\section{References}

[1] S.P. Martin, A supersymmetry primer, Adv. Ser. Direct. High Energy Phys. 18 (1998) 1 [Adv. Ser. Direct. High Energy Phys. 21 (2010) 1] [hep-ph/9709356] [INSPIRE].

[2] L. Randall and R. Sundrum, A large mass hierarchy from a small extra dimension, Phys. Rev. Lett. 83 (1999) 3370 [hep-ph/9905221] [INSPIRE].

[3] D. Stancato and J. Terning, The Unhiggs, JHEP 11 (2009) 101 [arXiv:0807.3961] [INSPIRE].

[4] D. Stancato and J. Terning, Constraints on the Unhiggs model from top quark decay, Phys. Rev. D 81 (2010) 115012 [arXiv: 1002.1694] [INSPIRE].

[5] A. Falkowski and M. Pérez-Victoria, Holographic Unhiggs, Phys. Rev. D 79 (2009) 035005 [arXiv: 0810.4940] [INSPIRE].

[6] J.A. Cabrer, G. von Gersdorff and M. Quirós, Soft-wall stabilization, New J. Phys. 12 (2010) 075012 [arXiv: 0907.5361] [INSPIRE].

[7] C. Englert, M. Spannowsky, D. Stancato and J. Terning, Unconstraining the Unhiggs, Phys. Rev. D 85 (2012) 095003 [arXiv:1203.0312] [InSPIRE].

[8] C. Englert, D.G. Netto, M. Spannowsky and J. Terning, Constraining the Unhiggs with LHC data, Phys. Rev. D 86 (2012) 035010 [arXiv:1205.0836] [InSPIRE].

[9] D. Gonçalves, T. Han and S. Mukhopadhyay, Higgs couplings at high scales, Phys. Rev. D 98 (2018) 015023 [arXiv: 1803.09751] [INSPIRE].

[10] C. Csáki, G. Lee, S.J. Lee, S. Lombardo and O. Telem, Continuum naturalness, JHEP 03 (2019) 142 [arXiv:1811.06019] [InSPIRE].

[11] S.J. Lee, M. Park and Z. Qian, Probing unitarity violation in the tail of the off-shell Higgs boson in $V_{L} V_{L}$ mode, Phys. Rev. D 100 (2019) 011702 [arXiv:1812.02679] [InSPIRE].

[12] E. Megías and M. Quirós, Gapped continuum Kaluza-Klein spectrum, JHEP 08 (2019) 166 [arXiv: 1905.07364] [INSPIRE].

[13] A. Shayegan Shirazi and J. Terning, Quantum critical Higgs: from AdS $S_{5}$ to colliders, JHEP 02 (2020) 026 [arXiv: 1908.06186] [INSPIRE].

[14] C. Gao, A. Shayegan Shirazi and J. Terning, Collider phenomenology of a gluino continuum, JHEP 01 (2020) 102 [arXiv: 1909.04061] [InSPIRE].

[15] H. Georgi, Unparticle physics, Phys. Rev. Lett. 98 (2007) 221601 [hep-ph/0703260] [INSPIRE].

[16] J. Alwall et al., The automated computation of tree-level and next-to-leading order differential cross sections, and their matching to parton shower simulations, JHEP 07 (2014) 079 [arXiv: 1405.0301] [INSPIRE].

[17] K. Agashe, A. Belyaev, T. Krupovnickas, G. Perez and J. Virzi, LHC signals from warped extra dimensions, Phys. Rev. D 77 (2008) 015003 [hep-ph/0612015] [INSPIRE].

[18] B. Lillie, L. Randall and L.-T. Wang, The bulk RS KK-gluon at the LHC, JHEP 09 (2007) 074 [hep-ph/0701166] [INSPIRE].

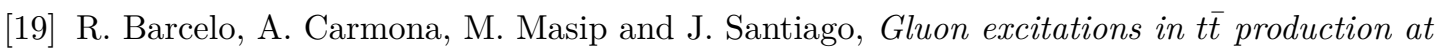
hadron colliders, Phys. Rev. D 84 (2011) 014024 [arXiv:1105.3333] [INSPIRE]. 
[20] R. Barcelo, A. Carmona, M. Masip and J. Santiago, Stealth gluons at hadron colliders, Phys. Lett. B 707 (2012) 88 [arXiv:1106.4054] [InSPIRE].

[21] S. Dasgupta, S.K. Rai and T.S. Ray, Impact of a colored vector resonance on the collider constraints for top-like top partner, Phys. Rev. D 102 (2020) 115014 [arXiv:1912.13022] [INSPIRE].

[22] D. Liu, L.-T. Wang and K.-P. Xie, Broad composite resonances and their signals at the LHC, Phys. Rev. D 100 (2019) 075021 [arXiv:1901.01674] [INSPIRE].

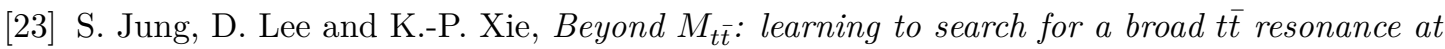
the LHC, Eur. Phys. J. C 80 (2020) 105 [arXiv:1906.02810] [InSPIRE].

[24] CMS collaboration, Search for production of four top quarks in final states with same-sign or multiple leptons in proton-proton collisions at $\sqrt{s}=13$ TeV, Eur. Phys. J. C 80 (2020) 75 [arXiv: 1908.06463] [INSPIRE].

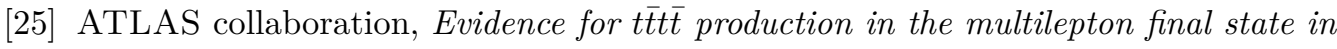
proton-proton collisions at $\sqrt{s}=13 \mathrm{TeV}$ with the ATLAS detector, Eur. Phys. J. C 80 (2020) 1085 [arXiv: 2007.14858] [INSPIRE].

[26] CMS collaboration, Measurement of the Higgs boson production rate in association with top quarks in final states with electrons, muons, and hadronically decaying tau leptons at $\sqrt{s}=13$ TeV, Eur. Phys. J. C 81 (2021) 378 [arXiv:2011.03652] [InSPIRE].

[27] ATLAS collaboration, Analysis of $t \bar{t} H$ and $t \bar{t} W$ production in multilepton final states with the ATLAS detector, Tech. Rep. ATLAS-CONF-2019-045, CERN, Geneva, Switzerland (2019).

[28] CMS collaboration, Measurement of top quark pair production in association with a $Z$ boson in proton-proton collisions at $\sqrt{s}=13 \mathrm{TeV}$, JHEP 03 (2020) 056 [arXiv:1907.11270] [INSPIRE].

[29] ATLAS collaboration, Measurements of the inclusive and differential production cross sections of a top-quark-antiquark pair in association with a $Z$ boson at $\sqrt{s}=13 \mathrm{TeV}$ with the ATLAS detector, Tech. Rep. ATLAS-CONF-2020-028, CERN, Geneva, Switzerland (2020).

[30] W.D. Goldberger and M.B. Wise, Modulus stabilization with bulk fields, Phys. Rev. Lett. 83 (1999) 4922 [hep-ph/9907447] [INSPIRE].

[31] O. DeWolfe, D.Z. Freedman, S.S. Gubser and A. Karch, Modeling the fifth-dimension with scalars and gravity, Phys. Rev. D 62 (2000) 046008 [hep-th/9909134] [InSPIRE].

[32] K. Agashe, A. Delgado, M.J. May and R. Sundrum, RS1, custodial isospin and precision tests, JHEP 08 (2003) 050 [hep-ph/0308036] [INSPIRE].

[33] M. Carena, E. Megías, M. Quíros and C. Wagner, $R_{D^{(*)}}$ in custodial warped space, JHEP 12 (2018) 043 [arXiv : 1809.01107] [InSPIRE].

[34] R. Contino, Y. Nomura and A. Pomarol, Higgs as a holographic pseudo-Goldstone boson, Nucl. Phys. B 671 (2003) 148 [hep-ph/0306259] [INSPIRE].

[35] K. Agashe, R. Contino and A. Pomarol, The minimal composite Higgs model, Nucl. Phys. B 719 (2005) 165 [hep-ph/0412089] [INSPIRE].

[36] R. Contino, L. Da Rold and A. Pomarol, Light custodians in natural composite Higgs models, Phys. Rev. D 75 (2007) 055014 [hep-ph/0612048] [INSPIRE]. 
[37] J.A. Cabrer, G. von Gersdorff and M. Quirós, Warped electroweak breaking without custodial symmetry, Phys. Lett. B 697 (2011) 208 [arXiv:1011.2205] [INSPIRE].

[38] J.A. Cabrer, G. von Gersdorff and M. Quirós, Suppressing electroweak precision observables in 5D warped models, JHEP 05 (2011) 083 [arXiv:1103.1388] [INSPIRE].

[39] J.A. Cabrer, G. von Gersdorff and M. Quirós, Improving naturalness in warped models with a heavy bulk Higgs boson, Phys. Rev. D 84 (2011) 035024 [arXiv:1104.3149] [INSPIRE].

[40] A. Carmona, E. Ponton and J. Santiago, Phenomenology of non-custodial warped models, JHEP 10 (2011) 137 [arXiv:1107.1500] [INSPIRE].

[41] J.A. Cabrer, G. von Gersdorff and M. Quirós, Flavor phenomenology in general $5 D$ warped spaces, JHEP 01 (2012) 033 [arXiv:1110.3324] [INSPIRE].

[42] M. Quirós, Higgs bosons in extra dimensions, Mod. Phys. Lett. A 30 (2015) 1540012 [arXiv: 1311.2824] [INSPIRE].

[43] J. de Blas, A. Delgado, B. Ostdiek and A. de la Puente, LHC signals of non-custodial warped 5 D models, Phys. Rev. D 86 (2012) 015028 [arXiv:1206.0699] [InSPIRE].

[44] E. Megias, O. Pujolàs and M. Quirós, On dilatons and the LHC diphoton excess, JHEP 05 (2016) 137 [arXiv: 1512.06106] [INSPIRE].

[45] E. Megias, G. Panico, O. Pujolàs and M. Quirós, A natural origin for the LHCb anomalies, JHEP 09 (2016) 118 [arXiv: 1608.02362] [INSPIRE].

[46] E. Megias, M. Quirós and L. Salas, Lepton-flavor universality violation in $R_{K}$ and $R_{D^{(*)}}$ from warped space, JHEP 07 (2017) 102 [arXiv: 1703.06019] [INSPIRE].

[47] A. Falkowski, M. González-Alonso and K. Mimouni, Compilation of low-energy constraints on 4-fermion operators in the SMEFT, JHEP 08 (2017) 123 [arXiv:1706. 03783] [INSPIRE].

[48] S. Willenbrock and G. Valencia, On the definition of the $Z$ boson mass, Phys. Lett. B 259 (1991) 373 [INSPIRE].

[49] T. Bhattacharya and S. Willenbrock, Particles near threshold, Phys. Rev. D 47 (1993) 4022 [INSPIRE].

[50] R. Escribano, A. Gallegos, J.L. Lucio M, G. Moreno and J. Pestieau, On the mass, width and coupling constants of the $f_{0}(980)$, Eur. Phys. J. C 28 (2003) 107 [hep-ph/0204338] [INSPIRE].

[51] E. Eichten, I. Hinchliffe, K.D. Lane and C. Quigg, Super collider physics, Rev. Mod. Phys. 56 (1984) 579 [Addendum ibid. 58 (1986) 1065] [INSPIRE].

[52] ATLAS collaboration, Search for heavy particles decaying into a top-quark pair in the fully hadronic final state in pp collisions at $\sqrt{s}=13 \mathrm{TeV}$ with the ATLAS detector, Phys. Rev. D 99 (2019) 092004 [arXiv: 1902.10077] [InSPIRE].

[53] R. Frederix, D. Pagani and M. Zaro, Large NLO corrections in $t \bar{t} W^{ \pm}$and $t \bar{t} t \bar{t}$ hadroproduction from supposedly subleading EW contributions, JHEP 02 (2018) 031 [arXiv: 1711.02116] [INSPIRE]. 